\title{
Eigenvalues of strings and cylinders with variable mass density
}

\author{
RICHARD SNYDER LAUGESEN ${ }^{1}$
}

\section{Introduction.}

This paper proves sharp inequalities for the frequencies of vibration of strings and cylindrical membranes, under either fixed or fixed-free boundary conditions. We establish such inequalities also on the "generalized" cylinder, which is the cross-product of an interval with a homogeneous Riemannian manifold. The goal is to better understand the effect that inhomogeneities in the mass distribution have on the frequencies of vibration.

Theorem 2, for example, says roughly that for a vibrating string fixed at one end and free at the other, moving mass towards the free end will tend to decrease the frequencies. To make this more precise, suppose $w$ is a positive function on the interval $(0, L)$ and $\lambda_{j}(w)$ is the $j$-th eigenvalue of the weighted Laplacian $w^{-1} d^{2} / d x^{2}$ on that interval, under Neumann boundary conditions at $x=0$ and Dirichlet boundary conditions at $x=L$; this means that for some eigenfunction $\psi_{j}$,

$$
\psi_{j}^{\prime \prime}+\lambda_{j}(w) w \psi_{j}=0 \quad \text { for } x \in(0, L), \quad \psi_{j}^{\prime}(0)=\psi_{j}(L)=0 .
$$

Physically, $\lambda_{j}(w)^{1 / 2}$ is proportional to the $j$-th characteristic frequency of a string with mass density $w$ that is free at the lefthand end and fixed at the right. The precise statement of Theorem 2 is that if the total mass $\int_{0}^{s} w(x) d x$ in each subinterval $(0, s)$ is at least $s$, then

$$
\sum_{j=1}^{n} \Phi\left(\frac{1}{\lambda_{j}(w)}\right) \geq \sum_{j=1}^{n} \Phi\left(\frac{1}{\lambda_{j}(1)}\right)
$$

for all positive integers $n$ and all convex increasing $\Phi$. Here $\lambda_{j}(1)$ is the $j$-th eigenvalue of the unweighted Laplacian $d^{2} / d x^{2}$, that is, the $j$-th eigenvalue

\footnotetext{
${ }^{1}$ Research partially supported by National Science Foundation Grant DMS9622837.
} 
for the homogeneous string with mass density $w \equiv 1$. Note that taking $\Phi(a)=a^{p}$ for $p \geq 1$ yields a result for the spectral zeta function, and that taking $n=1$ gives $\lambda_{1}(w) \leq \lambda_{1}(1)$, which means that moving mass towards the free end of the string certainly decreases the fundamental tone. Of course, this movement of mass might increase the $j$-th frequency for $j>1$, if we unwisely choose to move mass towards a nodal point of the corresponding eigenfunction of the homogeneous string. Thus it seems impossible to get a simple inequality that holds for each $\lambda_{j}$ individually, which is why we resort to "averaging" the eigenvalues by summing their reciprocals, in (1.1).

The rest of this introduction provides context for "isoperimetric" eigenvalue inequalities of this kind. First, it is intuitively clear that if we make a string (or membrane) heavier, it will vibrate more slowly. It is reasonable to suppose, also, that we can slow down the vibration by simply moving some of the mass of the string from places where the string vibrates very little towards places where it vibrates a lot, while not increasing the total mass. A result along these lines was proved by P.R. Beesack and B. Schwarz [4], who showed that for the interval $(-L, L)$ of length $2 L$ fixed at both ends, one has $\lambda_{1}(w) \leq \lambda_{1}\left(w_{*}\right)$, where $w_{*}$ is the symmetric increasing rearrangement of $w$; note that on each subinterval $(-s, s)$, the string with density $w$ has more mass than the string with density $w_{*}$, and so the $w$-string "should" vibrate more slowly. B. Schwarz [26, Theorem 1] extended this result to circular membranes, and Z. Nehari [23, Theorem II] found a similar result for superharmonic $w$; see [17, Theorem 4] for a generalization of these results.

The first eigenvalue is fairly well understood, then, but it is not clear how the higher eigenvalues should be affected by this shifting of mass around the membrane, since the higher eigenfunctions have complicated patterns of nodal lines. In general we cannot hope that a single mass density exists for which all the eigenvalues are extremal, and so we average the higher eigenvalues in order to get an extremal result. Two such averages for which results are already known are the spectral zeta function

$$
\sum_{j=1}^{\infty} \frac{1}{\lambda_{j}(w)^{p}}
$$

for fixed exponent $p$ and, more generally, the $\Phi$-functional

$$
\sum_{j=1}^{n} \Phi\left(\frac{1}{\lambda_{j}(w)}\right)
$$

for positive integers $n$ and convex increasing functions $\Phi$. (Take $\Phi(a)=a^{p}$ to recover the zeta function from the $\Phi$-functional.) In [18], for example, 
the author and C. Morpurgo studied the $\Phi$-functional for simply (and doubly) connected membranes under Dirichlet boundary conditions, with the extremals being obtained by conformally mapping the membranes to disks (and annuli). The $\Phi$-functional was shown to be convex in $w$, also. The author's paper [17] deals with inhomogeneous circular membranes with mass concentrated towards the center and develops extremal results for the spectral zeta function, results that fail for the $\Phi$-functional.

Incidentally, in two dimensions the operator $w^{-1} \Delta$ is exactly the Laplace-Beltrami operator for the metric $w g$ (where $g$ denotes the euclidean metric), and so our results for cylinders fall readily into spectral geometry. See the papers of S.-Y. A. Chang [9] and B. Osgood, R. Phillips and P. Sarnak [24] for somewhat related results on determinants of Laplacians, on various manifolds.

This paper proves only lower bounds on $1 / \lambda_{j}$, or equivalently, upper bounds on $\lambda_{j}$. Few sharp bounds in the opposite direction are known; most famous is surely the Faber-Krahn estimate $\lambda_{1}(\Omega) \geq \lambda_{1}\left(\Omega^{*}\right)$ for the fundamental tone of a domain $\Omega$ under Dirichlet boundary conditions (with $\Omega^{*}$ being the ball of the same volume as $\Omega$ ). J. M. Luttinger [19] extended the Faber-Krahn estimate to the trace of the heat kernel by showing that $\sum_{j=1}^{\infty} e^{-t \lambda_{j}\left(\Omega^{*}\right)} \geq \sum_{j=1}^{\infty} e^{-t \lambda_{j}(\Omega)}$ for all $t>0$. For further sharp estimates on eigenvalues, see the books by G. Pólya and G. Szegö [25] and C. Bandle [2], and the survey paper [1] by M. S. Ashbaugh and R. D. Benguria.

The next section presents results under fixed-free boundary conditions, and Section 3 does the same for the fixed boundary conditions. Then Section 4 describes open problems and conjectures, in particular for the trace of the heat kernel. See the Appendix for summary tables of the results in this paper and in [17], along with some discussion of the relative strengths of the three "mass concentration" hypotheses we use.

My thanks go to the Columbia University Mathematics Department for its hospitality during the summer of 1997, and to William Minicozzi for a helpful conversation.

\section{Results for mixed Dirichlet-Neumann boundary conditions.}

Fix $L>0$ throughout this section. 


\section{Fixed-free strings.}

Take a bounded positive function on the interval $(0, L)$, that is, take $w \in$ $\mathcal{L}^{\infty}(0, L)$ with $w>0$ a.e. Consider the eigenvalue problem with mixed Dirichlet-Neumann boundary conditions:

$$
-\psi^{\prime \prime}(x)=\lambda w(x) \psi(x) \quad \text { for } 0<x<L, \text { and } \quad \psi^{\prime}(0)=\psi(L)=0 .
$$

Physically, we think of the interval $(0, L)$ as representing an inhomogeneous string fixed at the righthand end and free at the left, and having mass density $w(x)$. The eigenvalues $\lambda$ give the squares of the frequencies of the string's modes of vibration. In this eigenvalue problem, the operator $-w^{-1} d^{2} / d x^{2}$ on $(0, L)$ is positive and has a discrete spectrum $\left\{\lambda_{j}(w)\right\}$, with $0<\lambda_{1}(w)<$ $\lambda_{2}(w) \leq \lambda_{3}(w) \leq \cdots \rightarrow \infty$. See Section 5 for further properties of the eigenvalues and eigenfunctions.

Our first theorem says that if we redistribute mass towards the free end of the string then the fundamental tone $\lambda_{1}$ goes down, exactly as intuition would suggest. Note that we do not need pointwise control of the mass density, just control of its integral.

Theorem 1. Take $w, v \in \mathcal{L}^{\infty}(0, L)$, with $w>0$ and $v>0$ a.e. and

$$
\int_{0}^{s} w(x) d x \geq \int_{0}^{s} v(x) d x \quad \text { for all } s \in(0, L) .
$$

Then

$$
\lambda_{1}(w) \leq \lambda_{1}(v)
$$

with strict inequality unless $w=v$ a.e.

See Section 7 for the proof, and [17, Theorem 4] for a similar theorem on the ball with Dirichlet boundary conditions. The hypothesis (2.1) says that the string with density $w$ has more mass in each interval $(0, s)$ near the free end of the string than does the string with mass density $v$.

The theorem does not extend to the second eigenvalue: one can take $v \equiv 1$ and apply perturbation methods to show that a function $w$ exists with $\int_{0}^{s} w(x) d x \geq s$ for all $s$ but with $\lambda_{2}(w)>\lambda_{2}(1)$. This makes sense: imagine taking mass from around the maximum point at $x=2 L / 3$ of the second mode $\psi_{2}(x)=-\cos (3 \pi x / 2 L)$ for the string of density 1 and moving the mass leftwards, placing the mass near the node of vibration at $x=L / 3$ and thus allowing the frequency to increase. 
The theorem does extend to the higher eigenvalues in an average sense, when $v \equiv 1$. Specifically, we will examine sums of finitely many reciprocal eigenvalues and convex means of these reciprocals; the spectral zeta function will be a special case. In order to state these extensions we need to develop some properties of the higher eigenvalues. Before doing so, though, we remark that the reciprocal $1 / \lambda_{j}$ is a reasonably natural object to consider since it is precisely the $j$-th eigenvalue for the inverse of the Laplacian (that is, the Green operator).

The first property we need is a lower bound of Weyl type: for some $\alpha \in(0,1)$ that depends on $w$,

$$
\lambda_{j}(w) \geq \alpha j^{2} \quad \text { for all } j \geq 1,
$$

which is a special case of (5.3). From this it follows that we may define the zeta function of the operator $w^{-1} d^{2} / d x^{2}$ on $(0, L)$ to be

$$
\sum_{j=1}^{\infty} \frac{1}{\lambda_{j}(w)^{p}} \quad \text { for } p>\frac{1}{2}
$$

The zeta function derives its name from its similarity to the Riemann zeta function. We also define the $\Phi$-functional

$$
\sum_{j=1}^{n} \Phi\left(\frac{1}{\lambda_{j}(w)}\right)
$$

for convex increasing $\Phi$ and $n$ either a positive integer or $+\infty$. Obviously this gives the zeta function when $n=+\infty$ and $\Phi(a)=a^{p}$ for fixed $p \geq 1$.

The monotonicity principle for the $\Phi$-functional says that:

decreasing the function $w$ pointwise will increase every eigenvalue, and hence will decrease the $\Phi$-functional.

For suppose $w$ and $v$ are positive functions with $w \geq v$. Then obviously the minimax principle (5.1) implies that $\lambda_{j}(w) \leq \lambda_{j}(v)$ for all $j$, and this proves the monotonicity principle, since $\Phi$ is increasing.

The next theorem weakens the hypotheses of the monotonicity principle in the case that $v \equiv 1$, by showing that the monotonicity principle holds provided only that the average of $w$ is at least 1 on every subinterval starting at the free end of the string. 
Theorem 2. Take $w \in \mathcal{L}^{\infty}(0, L)$, with $w>0$ a.e. and

$$
\int_{0}^{s} w(x) d x \geq s \quad \text { for all } s \in(0, L) .
$$

Let $n$ be a positive integer or $+\infty$. Let $\Phi(a)$ be convex and increasing for $a \geq 0$, with $\Phi(0)=0$ and $\Phi\left(\lambda_{1}(w)^{-1}\right)>0$. Then

$$
\sum_{j=1}^{n} \Phi\left(\frac{1}{\lambda_{j}(w)}\right) \geq \sum_{j=1}^{n} \Phi\left(\frac{1}{\lambda_{j}(1)}\right)
$$

with strict inequality unless $w=1$ a.e.

\section{Corollary 3.}

$$
\sum_{j=1}^{\infty} \frac{1}{\lambda_{j}(w)^{p}} \geq \sum_{j=1}^{\infty} \frac{1}{\lambda_{j}(1)^{p}}=\left(\frac{L}{\pi}\right)^{2 p}\left(2^{2 p}-1\right) \zeta(2 p) \quad \text { for all } p \geq 1
$$

with strict inequality unless $w=1$ a.e. (Here $\zeta$ is the Riemann zeta function.)

Both theorem and corollary will be proved in Section 8. Note that $\lambda_{j}(1)=$ $(2 j-1)^{2}(\pi / 2 L)^{2}$; we give the corresponding (trigonometric) eigenfunctions explicitly in Section 8.

Very roughly speaking, the theorem says that concentrating the mass near the free end of the string will tend to decrease the frequencies of vibration. For example, we can choose $\Phi(a)=a$ in Theorem 2, and with $n=1$ this choice gives $\lambda_{1}(w) \leq \lambda_{1}(1)$, which is a special case of Theorem 1 above. In addition, see Section 4 for remarks on the choice $\Phi(a)=e^{-t / a}$ (which is convex for small $a$ ) and its relation to the trace of the heat kernel.

The assumption in the theorem that $\Phi\left(\lambda_{1}(w)^{-1}\right)>0$ rules out the trivial case where both sides of (2.4) equal zero. That trivial case would have ruined the theorem's "strict inequality" statement. Also, note that the hypothesis (2.3) certainly holds if $w(x)$ is decreasing and $\int_{0}^{L} w d x \geq L$.

The conclusion (2.5) need not be true for $p$ near $1 / 2$; cf. [17, §3].

\section{Fixed-free cylinders.}

Write $\mathcal{C}:=(0, L) \times S^{1}$ for the cylinder of radius 1 and length $L>0$, and take a bounded positive function $w$ on the cylinder: $w \in \mathcal{L}^{\infty}(\mathcal{C})$ and $w>0$ a.e., with respect to Lebesgue measure $d x d \theta$ on $\mathcal{C}$. Consider the following 
eigenvalue problem on the cylinder with Neumann boundary conditions at the lefthand end and Dirichlet conditions at the righthand end:

$$
-\Delta \psi=\lambda w \psi \quad \text { for } 0<x<L \text { and all } \theta,
$$

and

$$
\frac{\partial \psi}{\partial x}(0, \theta)=\psi(L, \theta)=0
$$

Here

$$
\Delta=\frac{\partial^{2}}{\partial x^{2}}+\frac{\partial^{2}}{\partial \theta^{2}}
$$

denotes the usual Laplacian on the cylinder. Physically, we think of the cylinder as an inhomogeneous membrane fixed at the righthand end and free at the left, and having mass density $w(x, \theta)$. Note that $\lambda_{j}(w) \geq \alpha j$ by (5.3), and so the zeta function $\sum_{j=1}^{\infty} \lambda_{j}(w)^{-p}$ of the operator $w^{-1} \Delta$ on the cylinder makes sense for $p>1$. For a more detailed development of the eigenvalue problem, see Section 5.

The next theorem is the analogue for the cylinder of Theorem 2 for the inhomogeneous string, and we prove it in Section 9. Most of the discussion around Theorem 2 remains pertinent here.

Theorem 4. Take $w \in \mathcal{L}^{\infty}\left((0, L) \times S^{1}\right)$, with $w>0$ a.e. and

$$
\int_{0}^{s} \int_{0}^{2 \pi} w(x, \theta) d \theta d x \geq 2 \pi s \quad \text { for all } s \in(0, L) .
$$

Let $n$ be a positive integer or $+\infty$. Let $\Phi(a)$ be convex and increasing for $a \geq 0$, with $\Phi(0)=0, \Phi\left(\lambda_{1}(w)^{-1}\right)>0$ and $\int_{1}^{n} \Phi(1 / a)$ da finite. Then

$$
\sum_{j=1}^{n} \Phi\left(\frac{1}{\lambda_{j}(w)}\right) \geq \sum_{j=1}^{n} \Phi\left(\frac{1}{\lambda_{j}(1)}\right)
$$

with strict inequality unless $\int_{0}^{2 \pi} w(x, \theta) d \theta=2 \pi$ for almost all $x \in(0, L)$. If in addition $\Phi(a)$ is strictly convex, then (2.6) holds with strict inequality unless $w=1$ a.e.

Of course, when $w \equiv 1$ we have eigenvalues

$$
\left\{\lambda_{j}(1): j \geq 1\right\}=\left\{k^{2}+(2 \ell-1)^{2}(\pi / 2 L)^{2}: k \in \mathbf{Z}, \ell \geq 1\right\},
$$


by separation of variables. See Section 9 for this. Also, see Section 4 for comments on the choice $\Phi(a)=e^{-t / a}$, in relation to the trace of the heat kernel. Lastly, note that [16, Theorem 6] builds on work of J. Hersch [14, p. 32] to prove a result that has flavor similar to Theorem 4 but that does not require any assumptions on the distribution of mass.

\section{A computational result for a particular generalized cylinder.}

The preceding subsections concern the fixed-free string $(0, L)$ and cylin$\operatorname{der}(0, L) \times S^{1}$, and give results for both the first eigenvalue (Theorem 1) and the $\Phi$-functional (Theorems 2 and 4 ). Naturally one wonders whether these results can be generalized to $(0, L) \times M$, for more general Riemannian manifolds $M$. For the first eigenvalue the results do indeed generalize: see Theorem 8 below. For the $\Phi$-functional the results need not successfully generalize, as we will see for the torus $M=S^{1} \times S^{1}$. This will involve looking at the first 53,199 eigenvalues of $(0, \pi / 2) \times S^{1} \times S^{1}$. Note that we investigate only for length $L=\pi / 2$, though for other values of $L$ a similar approach should be possible.

Write

$$
\mathcal{D}:=(0, \pi / 2) \times S^{1} \times S^{1}
$$

for the cross product of the interval of length $\pi / 2$ with the torus, and take a bounded positive function $w \in \mathcal{L}^{\infty}(\mathcal{D})$ with $w>0$ a.e. (with respect to Lebesgue measure $d x d \theta_{1} d \theta_{2}$ ). Consider the following eigenvalue problem on $\mathcal{D}$ with Neumann boundary conditions at the lefthand end $(x=0)$ and Dirichlet conditions at the righthand end $(x=\pi / 2)$ :

$$
-\Delta \psi=\lambda w \psi \quad \text { for }\left(x, \theta_{1}, \theta_{2}\right) \in \mathcal{D},
$$

and

$$
\frac{\partial \psi}{\partial x}\left(0, \theta_{1}, \theta_{2}\right)=\psi\left(\pi / 2, \theta_{1}, \theta_{2}\right)=0
$$

Here

$$
\Delta=\frac{\partial^{2}}{\partial x^{2}}+\frac{\partial^{2}}{\partial \theta_{1}^{2}}+\frac{\partial^{2}}{\partial \theta_{2}^{2}}
$$

denotes the Laplacian on $\mathcal{D}$. For a fuller description of the properties of the eigenvalues $\lambda_{j}(w)$, see Section 5 .

The next result is a partial analogue for $\mathcal{D}$ of Theorem 2 for the string and Theorem 4 for the cylinder. We will prove it rigorously for $n \leq 119$ and numerically for $n \leq 53055$ 
Computational Result 5. Take $w \in \mathcal{L}^{\infty}(\mathcal{D})$, with $w>0$ a.e. and

$$
\int_{0}^{s} \int_{0}^{2 \pi} \int_{0}^{2 \pi} w\left(x, \theta_{1}, \theta_{2}\right) d \theta_{1} d \theta_{2} d x \geq(2 \pi)^{2} s \quad \text { for all } s \in(0, \pi / 2) .
$$

Let $n$ be a positive integer with $1 \leq n \leq 53055$. Let $\Phi(a)$ be convex and increasing for $a \geq 0$, with $\Phi\left(\lambda_{1}(w)^{-1}\right)>\Phi(0)$. Then

$$
\sum_{j=1}^{n} \Phi\left(\frac{1}{\lambda_{j}(w)}\right) \geq \sum_{j=1}^{n} \Phi\left(\frac{1}{\lambda_{j}(1)}\right)
$$

with strict inequality unless $\int_{0}^{2 \pi} \int_{0}^{2 \pi} w\left(x, \theta_{1}, \theta_{2}\right) d \theta_{1} d \theta_{2}=(2 \pi)^{2}$ for almost all $x \in(0, \pi / 2)$. If in addition $\Phi(a)$ is strictly convex, then (2.8) holds with strict inequality unless $w=1$ a.e.

We discuss the theoretical and numerical evidence for this computational result in Section 10. We will reduce the question to showing positivity of 1370 trigonometric functions for $x \in(0, \pi)$. Numerical work strongly supports this positivity claim, but this falls short of a rigorous proof. For $1 \leq n \leq 119$, though, we do prove Computational Result 5 rigorously.

The number 53055 is not necessarily the largest number for which the result holds, but it is close to being optimal because a function $w \in \mathcal{L}^{\infty}(\mathcal{D})$ exists with $w>0$ a.e. such that $w$ satisfies (2.7) but

$$
\sum_{j=1}^{53199} \frac{1}{\lambda_{j}(w)}<\sum_{j=1}^{53199} \frac{1}{\lambda_{j}(1)} .
$$

For this we need only show that a certain trigonometric function is negative at $x=0.446 \pi$, which we do numerically. (See Section 10.)

The numbers appearing in the above results are related by the fact that

$$
1370=\lambda_{53055}(1)<\lambda_{53056}(1)=\cdots=\lambda_{53199}(1)=1371 .
$$

To check (2.10) by computer, it helps to recall that by separation of variables, the generalized cylinder $\mathcal{D}$ with mass density 1 has eigenvalues

$$
\left\{\lambda_{j}(1): j \geq 1\right\}=\left\{k_{1}^{2}+k_{2}^{2}+(2 \ell-1)^{2}: k_{1}, k_{2} \in \mathbf{Z}, \ell \geq 1\right\}
$$

(listing with multiplicity). 


\section{Generalized cylinders with mixed boundary conditions.}

By strengthening the hypotheses in the preceding results, we obtain theorems valid for all $n$ and for a much wider class of product manifolds.

Definition. Call a Riemannian manifold $M_{g}$ homogeneous if its isometry group is transitive, meaning that for each $x, y \in M$ an isometry $\tau$ of $M_{g}$ exists with $\tau(x)=y$.

Let $M_{g}$ be a compact, connected, homogeneous Riemannian manifold of dimension $N \geq 1$. For example, $M_{g}$ could be the circle, sphere, torus or real projective space, or it could be a compact symmetric space, a compact connected Lie group, or a compact quotient $G / H$ in which $G$ is a connected Lie group with compact Lie subgroup $H$ (see [6, pp. 247-251, 351-353]). Write $d V$ for the volume element on $M_{g}$ and $V(M)$ for the total volume of $M$. The eigenvalue problem on the generalized cylinder $(0, L) \times M$ is

$$
-\Delta \psi=\lambda w \psi \quad \text { for }(x, \theta) \in(0, L) \times M
$$

and

$$
\frac{\partial \psi}{\partial x}(0, \theta)=\psi(L, \theta)=0 \text { for all } \theta \in M
$$

Here $\Delta=\partial^{2} / \partial x^{2}+\Delta_{g}$, where $\Delta_{g}$ is the Laplace-Beltrami operator on $M_{g}$. The operator $-w^{-1} \Delta$ on $(0, L) \times M$ is positive and has a discrete spectrum $\left\{\lambda_{j}(w)\right\}$, with $0<\lambda_{1}(w)<\lambda_{2}(w) \leq \lambda_{3}(w) \leq \cdots \rightarrow \infty$. Section 5 details the properties of these eigenvalues, in particular the lower bound of Weyl type $\lambda_{j}(w) \geq \alpha j^{2 /(1+N)}$, which implies that the zeta function of the eigenvalues makes sense for $p>(1+N) / 2$.

In almost every one of our results so far, the extremal case has been when $w \equiv 1$. More generally, one can try to establish results in which the extremal case occurs when $w$ is some radial mass density function, not necessarily constant. The next result has this form.

Theorem 6. Take $w, v \in \mathcal{L}^{\infty}((0, L) \times M)$, with $w>0$ and $v>0$ a.e. and with $v$ independent of $\theta \in M$, so that $v=v(x)$. Assume

$$
\int_{M} w(x, \theta) d V(\theta) \geq V(M) v(x) \quad \text { for almost all } x \in(0, L) \text {. }
$$


Let $n$ be either a positive integer or $+\infty$. Let $\Phi(a)$ be convex and increasing for $a \geq 0$, with $\Phi(0)=0, \Phi\left(\lambda_{1}(w)^{-1}\right)>0$ and $\int_{1}^{n} \Phi\left(1 / a^{2 /(1+N)}\right) d a$ finite. Then

$$
\sum_{j=1}^{n} \Phi\left(\frac{1}{\lambda_{j}(w)}\right) \geq \sum_{j=1}^{n} \Phi\left(\frac{1}{\lambda_{j}(v)}\right)
$$

with strict inequality unless $\int_{M} w(x, \theta) d V(\theta)=V(M) v(x)$ for almost all $x \in(0, L)$. If in addition $\Phi(a)$ is strictly convex, then (2.12) holds with strict inequality unless $w=v$ a.e.

In particular, averaging $w$ over $M$ decreases the $\Phi$-functional:

$$
\sum_{j=1}^{n} \Phi\left(\frac{1}{\lambda_{j}(w)}\right) \geq \sum_{j=1}^{n} \Phi\left(\frac{1}{\lambda_{j}(\bar{w})}\right)
$$

where $\bar{w}(x):=\int_{M} w(x, \theta) d V(\theta) / V(M)$.

The theorem is proved in Section 11; note that it extends [16, Theorem 1], which is essentially just the "cylinder" case $M=S^{1}$ of Theorem 6 .

The hypothesis (2.11) is fairly strong, but as the proof of the theorem makes clear, to weaken that hypothesis one would seem to need to know nontrivial properties of the eigenfunctions of $v^{-1} \Delta$.

\section{The generalized cylinder with decreasing mass density.}

The hypothesis (2.13) in the next theorem ensures rather strongly that the $w$-mass is concentrated towards the free end (where $x=0$ ); note that we also assume the total mass is at least $L \cdot V(M)$, which is the total mass of the homogeneous manifold with mass density 1 . These hypotheses are stronger than those in Theorems 2 and 4 and Computational Result 5.

Theorem 7. Take $w \in \mathcal{L}^{\infty}((0, L) \times M)$, with $\int_{0}^{L} \int_{M} w(x, \theta) d V(\theta) d x \geq$ $L \cdot V(M)$ and $w>0$ a.e. and with

$$
x \mapsto \int_{M} w(x, \theta) d V(\theta) \quad \text { decreasing for } x \in(0, L) .
$$

Let $n$ be either a positive integer or $+\infty$. Let $\Phi(a)$ be convex and increasing for $a \geq 0$, with $\Phi(0)=0, \Phi\left(\lambda_{1}(w)^{-1}\right)>0$ and $\int_{1}^{n} \Phi\left(1 / a^{2 /(1+N)}\right)$ da finite. Then

$$
\sum_{j=1}^{n} \Phi\left(\frac{1}{\lambda_{j}(w)}\right) \geq \sum_{j=1}^{n} \Phi\left(\frac{1}{\lambda_{j}(1)}\right)
$$


with strict inequality unless $\int_{M} w(x, \theta) d V(\theta)=V(M)$ for almost all $x \in$ $(0, L)$. If in addition $\Phi(a)$ is strictly convex, then (2.14) holds with strict inequality unless $w=1$ a.e.

We prove this theorem in Section 12.

To be careful, we know only that the integral $\int_{M} w(x, \theta) d V(\theta)$ appearing in (2.13) is defined for almost all $x$, and so our hypothesis should be interpreted as meaning that a decreasing function of $x$ exists that equals the integral in (2.13) for almost every $x \in(0, L)$.

The first eigenvalue of the fixed-free generalized cylinder.

We conclude the section with a comparison theorem for the first eigenvalue of the generalized cylinder $(0, L) \times M$, analogous to Theorem 1 for the string. See also [17, Theorem 4] for a similar theorem on the ball with fixed boundary conditions.

Theorem 8. Take $w, v \in \mathcal{L}^{\infty}((0, L) \times M)$ with $w>0$ and $v>0$ a.e. and with $v$ independent of $\theta \in M$, so that $v=v(x)$. If

$$
\int_{0}^{s} \int_{M} w(x, \theta) d V(\theta) d x \geq V(M) \int_{0}^{s} v(x) d x \quad \text { for all } s \in(0, L)
$$

then

$$
\lambda_{1}(w) \leq \lambda_{1}(v)
$$

with strict inequality unless $w=v$ a.e.

See Section 13 for the proof. Observe that our hypothesis (2.15) in this result is weaker than our hypotheses in Theorems 6 and 7 .

\section{Results for Dirichlet boundary conditions.}

In this section we briefly consider analogous eigenvalue problems for strings and cylinders with both ends fixed. The eigenfunctions are now required to equal zero on the entire boundary. (For a fuller treatment of the technical aspects, see Section 5.) Again we fix $L>0$, but now for convenience we consider the string $(-L, L)$ and cylinder $(-L, L) \times S^{1}$ of length $2 L$, fixed at $x= \pm L$.

For the string with Dirichlet boundary conditions, the analogues of Theorem 2 and Corollary 3 are: 
Theorem 9 ([17, Theorem 2]). Take $w \in \mathcal{L}^{\infty}(-L, L)$. Assume $w>0$ a.e. and

$$
\int_{-s}^{s} w(x) d x \geq 2 s \quad \text { for all } s \in(0, L) .
$$

Take $n$ to be either a positive integer or $+\infty$. Let $\Phi(a)$ be convex and increasing for $a \geq 0$, with $\Phi(0)=0$ and $\Phi\left(\lambda_{1}(w)^{-1}\right)>0$. Then

$$
\sum_{j=1}^{n} \Phi\left(\frac{1}{\lambda_{j}(w)}\right) \geq \sum_{j=1}^{n} \Phi\left(\frac{1}{\lambda_{j}(1)}\right)
$$

with strict inequality unless $w(x)+w(-x)=2$ a.e. If in addition $\Phi(a)$ is strictly convex, then (3.2) holds with strict inequality unless $w=1$ a.e.

Corollary $10([17, \S 2])$.

$$
\sum_{j=1}^{\infty} \frac{1}{\lambda_{j}(w)^{p}} \geq \sum_{j=1}^{\infty} \frac{1}{\lambda_{j}(1)^{p}}=\left(\frac{2 L}{\pi}\right)^{2 p} \zeta(2 p) \quad \text { for all } p>1,
$$

with strict inequality unless $w=1$ a.e. (Here $\zeta$ is the Riemann zeta function.)

Recall that for the homogeneous string, $\lambda_{j}(1)=(j \pi / 2 L)^{2}$, with the eigenfunction being trigonometric.

The hypothesis (3.1) says roughly that the $w$-mass is concentrated towards the middle of the string, away from the fixed endpoints. Intuitively we expect this to lower the frequencies of vibration, and that is what the theorem says, in an average sense.

The next result shows, however, that the preceding theorem for the fixed string does not carry over completely to the fixed cylinder, unlike the situation in Theorem 4 for the fixed-free cylinder, and instead holds only for $n \leq 4$. Essentially, Theorem 4 is better than Theorem 11 because sums of sines of odd multiples of $x$ are better behaved than sums of sines of all multiples (see Lemmas 15 and 16 in Section 6).

Theorem 11. Take $w \in \mathcal{L}^{\infty}\left((-L, L) \times S^{1}\right)$, with $w>0$ a.e. and

$$
\int_{-s}^{s} \int_{0}^{2 \pi} w(x, \theta) d \theta d x \geq 2 \pi \cdot 2 s \quad \text { for all } s \in(0, L) .
$$


Assume $1 \leq n \leq 4$. Let $\Phi(a)$ be convex and increasing for $a \geq 0$, with $\Phi\left(\lambda_{1}(w)^{-1}\right)>\Phi(0)$. Then

$$
\sum_{j=1}^{n} \Phi\left(\frac{1}{\lambda_{j}(w)}\right) \geq \sum_{j=1}^{n} \Phi\left(\frac{1}{\lambda_{j}(1)}\right)
$$

with strict inequality unless $\int_{0}^{2 \pi}[w(x, \theta)+w(-x, \theta)] d \theta=4 \pi$ for almost all $x \in(0, L)$. If in addition $\Phi(a)$ is strictly convex, then (3.3) holds with strict inequality unless $w=1$ a.e.

Furthermore, for each $n>4$, positive numbers $L_{1}(n)$ and $L_{2}(n)$ exist with $L_{1}(n)<L_{2}(n)$ such that the theorem still holds for $n$ provided $L \in\left(0, L_{1}(n)\right] \cup\left[L_{2}(n),+\infty\right)$. We see, then, that the problem lies with the middle range of cylinder lengths $L$, between $L_{1}(n)$ and $L_{2}(n)$; the theorem can indeed fail for these intermediate $L$ values, as can be seen (for $n=5$ ) by arguing along the lines of [17, Theorem 3].

We prove this theorem in Section 14, as well as showing there that we can take, for example, $L_{1}(5)=\sqrt{2} \pi / 2$ and $L_{2}(5)=\sqrt{5} \pi / 2$. Somewhat longer arguments show that we can take $L_{1}(6)=\pi / 2$ and $L_{2}(6)=\sqrt{5} \pi / 2$.

\section{Generalized cylinders with fixed boundary conditions.}

Again let $M_{g}$ be a compact, connected, homogeneous Riemannian manifold of dimension $N \geq 1$. The eigenvalue problem on $(-L, L) \times M$ is

$$
-\Delta \psi=\lambda w \psi \quad \text { for }(x, \theta) \in(-L, L) \times M,
$$

and

$$
\psi( \pm L, \theta)=0 \text { for all } \theta \in M
$$

Here $\Delta=\partial^{2} / \partial x^{2}+\Delta_{g}$, with $\Delta_{g}$ being the Laplace-Beltrami operator on $M_{g}$. Again Section 5 discusses the properties of these eigenvalues.

The next theorem is the analogue for Dirichlet boundary conditions of Theorem 6. Its statement differs from that of Theorem 6 only in writing $(-L, L)$ instead of $(0, L)$, but we state the theorem anyway so that we can refer clearly to it later on.

Theorem 12. Take $w, v \in \mathcal{L}^{\infty}((-L, L) \times M)$, with $w>0$ and $v>0$ a.e. and with $v$ independent of $\theta \in M$, so that $v=v(x)$. Assume

$$
\int_{M} w(x, \theta) d V(\theta) \geq V(M) v(x) \quad \text { for almost all } x \in(-L, L) .
$$


Let $n$ be either a positive integer or $+\infty$. Let $\Phi(a)$ be convex and increasing for $a \geq 0$, with $\Phi(0)=0, \Phi\left(\lambda_{1}(w)^{-1}\right)>0$ and $\int_{1}^{n} \Phi\left(1 / a^{2 /(1+N)}\right) d a$ finite. Then

$$
\sum_{j=1}^{n} \Phi\left(\frac{1}{\lambda_{j}(w)}\right) \geq \sum_{j=1}^{n} \Phi\left(\frac{1}{\lambda_{j}(v)}\right)
$$

with strict inequality unless $\int_{M} w(x, \theta) d V(\theta)=V(M) v(x)$ for almost all $x \in(-L, L)$. If in addition $\Phi(a)$ is strictly convex, then (3.5) holds with strict inequality unless $w=v$ a.e.

In particular, averaging $w$ over $M$ decreases the $\Phi$-functional:

$$
\sum_{j=1}^{n} \Phi\left(\frac{1}{\lambda_{j}(w)}\right) \geq \sum_{j=1}^{n} \Phi\left(\frac{1}{\lambda_{j}(\bar{w})}\right)
$$

where $\bar{w}(x):=\int_{M} w(x, \theta) d V(\theta) / V(M)$.

The theorem is proved in Section 15; note that it extends [18, Theorem 1(b)], which is essentially just the "cylinder" case $M=S^{1}$ of Theorem 12 .

The hypothesis (3.6) in the next theorem amounts to a strong assumption that the $w$-mass is concentrated towards the middle (where $x=0$ ). Note also that in the theorem we assume the total mass is at least $2 L \cdot V(M)$, which is the total mass of the homogeneous manifold with mass density 1.

Theorem 13. Take $w \in \mathcal{L}^{\infty}((-L, L) \times M)$, with $\int_{-L}^{L} \int_{M} w(x, \theta) d V(\theta) d x \geq$ $2 L \cdot V(M)$ and $w>0$ a.e. and with

$$
x \mapsto \int_{M}[w(x, \theta)+w(-x, \theta)] d V(\theta) \quad \text { decreasing for } x \in(0, L) .
$$

Let $n$ be either a positive integer or $+\infty$. Let $\Phi(a)$ be convex and increasing for $a \geq 0$, with $\Phi(0)=0, \Phi\left(\lambda_{1}(w)^{-1}\right)>0$ and $\int_{1}^{n} \Phi\left(1 / a^{2 /(1+N)}\right) d a$ finite. Then

$$
\sum_{j=1}^{n} \Phi\left(\frac{1}{\lambda_{j}(w)}\right) \geq \sum_{j=1}^{n} \Phi\left(\frac{1}{\lambda_{j}(1)}\right)
$$

with strict inequality unless $\int_{M}[w(x, \theta)+w(-x, \theta)] d V(\theta)=2 V(M)$ for almost all $x \in(0, L)$. If in addition $\Phi(a)$ is strictly convex, then (3.7) holds with strict inequality unless $w=1$ a.e. 
We prove this theorem in Section 16.

Being careful, we know only that the integral

$$
\int_{M}[w(x, \theta)+w(-x, \theta)] d V(\theta)
$$

appearing in (3.6) is defined for almost all $x$, and so our hypothesis should be interpreted as meaning that a decreasing function of $x$ exists that equals the integral in (3.6) for almost every $x \in(0, L)$.

Notice that the "decreasing mass" hypothesis (3.6) is automatically satisfied if $w$ is smooth and superharmonic (so that $\Delta w \leq 0$ ), since in that case the function in (3.6) is concave and even in $x$.

\section{The first eigenvalue of the fixed generalized cylinder.}

We finish with a comparison theorem for the first eigenvalue of the generalized cylinder $(-L, L) \times M$ under Dirichlet boundary conditions, analogous to Theorem 8 .

Theorem 14. Take $w, v \in \mathcal{L}^{\infty}((-L, L) \times M)$ with $w>0$ and $v>0$ a.e. and with $v$ independent of $\theta \in M$, so that $v=v(x)$. Assume $v$ is an even function of $x \in(-L, L)$. If

$$
\int_{-s}^{s} \int_{M} w(x, \theta) d V(\theta) d x \geq V(M) \int_{-s}^{s} v(x) d x \quad \text { for all } s \in(0, L)
$$

then

$$
\lambda_{1}(w) \leq \lambda_{1}(v)
$$

with strict inequality unless $w=v$ a.e.

See Section 17 for the proof. The hypothesis (3.8) says that the membrane with density $w$ has more mass near the middle $(x=0)$ than does the membrane with mass density $v$.

\section{Conjectures and questions.}

\section{The trace of the heat kernel.}

Many open questions revolve around the trace of the heat kernel, which is the functional $\sum_{j} e^{-\lambda_{j} t}$ (also known as the partition function). For example, 
do the extremal results for the $\Phi$-functional in Theorems 2, 4, 6, 7, 9, 12 and 13 hold also for the trace of the heat kernel? For Theorem 13, for example, the question is whether or not

$$
\sum_{j=1}^{\infty} e^{-\lambda_{j}(w) t} \geq \sum_{j=1}^{\infty} e^{-\lambda_{j}(1) t} \quad \text { for all } t>0
$$

under the hypotheses of Theorem 13.

Certainly these inequalities for the trace of the heat kernel are all true for $t \geq 2 / \lambda_{1}(1)$ (for $t \geq 2 / \lambda_{1}(v)$, in Theorems 6 and 12), simply by applying the corresponding theorems with $\Phi(a)=e^{-t / a}$, which is convex and increasing for $a \in(0, t / 2]$. For small time $t$, though, we proceed to show that the conjectured results for the trace of the heat kernel fail under mixed Neumann-Dirichlet boundary conditions, although they do hold for small time under purely Dirichlet boundary conditions.

Theorems 2 and 9 can fail for the trace of the heat kernel, for small time, by the same reasoning used in the " $N=1$ " case of $[17, \S 3]$ : the point is that the small time behavior of the trace of the heat kernel is dominated by a term $\int \sqrt{w(x)} d x / \sqrt{t}$.

Theorems 4, 6 and 7 can also fail for the trace of the heat kernel for small time, but here the reasoning is more subtle. Take $M=S^{1}$ and define

$$
w(x, \theta):=1+\frac{L-x}{2 L} \sin \theta, \quad v(x, \theta):=1,
$$

for $x \in[0, L], \theta \in \mathbf{R}$, so that $w$ and $v$ satisfy the hypotheses of Theorems 4, 6 and 7. The operator $w^{-1} \Delta$ equals the Laplace-Beltrami operator of the metric $w g$ on the cylinder $\mathcal{C}=(0, L) \times S^{1}$, where $g$ denotes the euclidean metric on the cylinder, and so putting $f \equiv 1$ in [7, Th.7.2] gives the asymptotic expansion

$$
\begin{aligned}
\sum_{j=1}^{\infty} e^{-\lambda_{j}(w) t}= & \frac{\operatorname{area}_{w g}(\mathcal{C})}{4 \pi t} \\
& +\frac{\text { length }_{w g}\left(\partial_{\mathrm{free}} \mathcal{C}\right)-\operatorname{length}_{w g}\left(\partial_{\mathrm{fixed}} \mathcal{C}\right)}{8 \sqrt{\pi t}}+O(1)
\end{aligned}
$$

as $t \rightarrow 0+$.

From our definitions of $w$ and $v$ we know that

$$
\operatorname{area}_{w g}(\mathcal{C})=\int_{0}^{L} \int_{0}^{2 \pi} w(x, \theta) d \theta d x=2 \pi L=\operatorname{area}_{v g}(\mathcal{C})
$$


and

$$
\text { length }_{w g}\left(\partial_{\text {free }} \mathcal{C}\right)=\int_{0}^{2 \pi} \sqrt{w(0, \theta)} d \theta<2 \pi=\operatorname{length}_{v g}\left(\partial_{\text {free }} \mathcal{C}\right)
$$

and

$$
\operatorname{length}_{w g}\left(\partial_{\text {fixed }} \mathcal{C}\right)=\int_{0}^{2 \pi} \sqrt{w(L, \theta)} d \theta=2 \pi=\operatorname{length}_{v g}\left(\partial_{\text {fixed }} \mathcal{C}\right)
$$

From (4.1) it follows that

$$
\sum_{j=1}^{\infty} e^{-\lambda_{j}(w) t}<\sum_{j=1}^{\infty} e^{-\lambda_{j}(v) t}
$$

for all small $t$, as we wished to show.

Of course, all this leaves open the possibility that Theorem 6 (respectively, Theorem 7) might be true for the trace of the heat kernel in higher dimensions, where $N \geq 2$. Note that for "nice" $w$, the first order asymptotic formula for the trace is

$$
\sum_{j=1}^{\infty} e^{-\lambda_{j}(w) t}=\frac{\int_{0}^{L} \int_{M} w(x, \theta)^{(1+N) / 2} d \theta d x}{(\text { const. }) t^{(1+N) / 2}}+O\left(t^{-N / 2}\right) \quad \text { as } t \rightarrow 0+
$$

and when $N \geq 2$ the integral on the righthand side is strictly minimal for $w \equiv v$ (respectively, $w \equiv 1$ ) by Hölder's inequality, in view of the hypotheses in the theorem.

For mixed boundary conditions, then, our conjectures about the trace are false. For purely Dirichlet boundary conditions, the situation seems more promising. Indeed, the analogues of Theorems 12 and 13 for the trace of the heat kernel are true for small time when $N \geq 2$ and are "almost known" to be true when $N=1$, as we now explain, and so it is reasonable to hope that they do hold for all time.

We assume $w$ and $v$ satisfy the hypotheses of Theorem 12 or Theorem 13; for Theorem 13 , we set $v \equiv 1$. In particular, then,

$$
\int_{-L}^{L} \int_{M} w(x, \theta) d V(\theta) d x \geq \int_{-L}^{L} \int_{M} v(x) d V(\theta) d x .
$$

For technical convenience, assume also that $w$ and $v$ are smooth and bounded away from zero. We assume $N=1$, since for $N \geq 2$ we can successfully 
argue like around (4.2). We want to show that

$$
\sum_{j=1}^{\infty} e^{-\lambda_{j}(w) t} \geq \sum_{j=1}^{\infty} e^{-\lambda_{j}(v) t} \quad \text { for all small } t>0
$$

Since $N=1$, the manifold $\mathcal{G}=(-L, L) \times M$ is two dimensional and the asymptotic formula for the trace of the heat kernel (under purely Dirichlet boundary conditions) is then

$$
\sum_{j=1}^{\infty} e^{-\lambda_{j}(w) t}=\frac{\operatorname{area}_{w g}(\mathcal{G})}{4 \pi t}-\frac{\text { length }_{w g}(\partial \mathcal{G})}{8 \sqrt{\pi t}}+O(1) \quad \text { as } t \rightarrow 0+
$$

which translates to:

$$
\begin{aligned}
\sum_{j=1}^{\infty} e^{-\lambda_{j}(w) t}= & \frac{\int_{-L}^{L} \int_{M} w(x, \theta) d V(\theta) d x}{4 \pi t} \\
& -\frac{\int_{M}[\sqrt{w(-L, \theta)}+\sqrt{w(L, \theta)}] d V(\theta)}{8 \sqrt{\pi t}}+O(1)
\end{aligned}
$$

as $t \rightarrow 0+$. We might as well assume equality holds in (4.3), since otherwise (4.5) shows that (4.4) is true. We will show that

$$
\begin{aligned}
& \int_{M}[\sqrt{w(-L, \theta)}+\sqrt{w(L, \theta)}] d V(\theta) \\
& \leq \int_{M}[\sqrt{v(-L)}+\sqrt{v(L)}] d V(\theta)
\end{aligned}
$$

If the inequality is strict then (4.4) will follow from (4.5), using the equality in (4.3). This is the sense in which the heat kernel results are "almost known" to be true for small time, when $N=1$. Of course, it is conceivable that when equality holds in (4.6), the heat kernel result (4.4) might fail for small time, but deciding this question would require a more detailed study of the asymptotics of the trace.

To prove (4.6) for $w$ as in Theorem 12, observe first that because of the 
equality in (4.3), equality must also hold in (3.4) for all $x$, and hence

$$
\begin{aligned}
& \int_{M} \sqrt{w( \pm L, \theta)} d V(\theta) \\
& \leq \sqrt{V(M)}\left(\int_{M} w( \pm L, \theta) d V(\theta)\right)^{1 / 2} \quad \text { by Cauchy-Schwarz } \\
& =V(M) \sqrt{v( \pm L)} \quad \text { by equality in (3.4) } \\
& =\int_{M} \sqrt{v( \pm L)} d V(\theta)
\end{aligned}
$$

which gives (4.6). To prove (4.6) for $w$ as in Theorem 13 and for $v \equiv 1$, note that

$$
\begin{aligned}
& \int_{M}[\sqrt{w(-L, \theta)}+\sqrt{w(L, \theta)}] d V(\theta) \\
& \leq 2 \int_{M} \sqrt{\frac{w(-L, \theta)+w(L, \theta)}{2}} d V(\theta) \quad \text { by concavity of } \sqrt{\cdot} \\
& \leq \sqrt{2 V(M)}\left(\int_{M}[w(-L, \theta)+w(L, \theta)] d V(\theta)\right)^{1 / 2} \quad \text { by Cauchy-Schwarz } \\
& \leq 2 V(M) \quad \text { by }(3.6) \text { and the equality in (4.3) } \\
& =\int_{M}[\sqrt{v(-L)}+\sqrt{v(L)}] d V(\theta),
\end{aligned}
$$

which is (4.6) again.

\section{Other directions.}

Few sharp upper bounds on the $\Phi$-functional, trace of the heat kernel or zeta function are currently known; this paper presents only lower bounds. See the remarks at the end of Section 1. Note that an upper bound for the $\Phi$-functional of the vibrating string $(N=0)$ was proved by D. Banks [3, Theorem 2.2], with the extremal mass density being a step function.

To read about known extremal results for the zeta function of the Laplacian and the trace of the heat kernel (on various manifolds), see $[16,17,18,19,21,22]$; also, H. L. Montgomery's paper [20] can be interpreted as extremizing the trace of the heat kernel for flat tori with a given area, and $[9,24]$ examine the determinant of the Laplacian. 


\section{Technical details.}

This section explains the properties of the eigenvalues and eigenfunctions that are needed in this paper. We work with the generalized cylinder, stating occasionally the simplifications that can be enjoyed for the cases of the string and cylinder.

\section{Mixed Dirichlet-Neumann boundary conditions.}

Fix $L>0$. Let $M_{g}$ be a compact, connected, homogeneous Riemannian manifold of dimension $N \geq 1$. Define the generalized cylinder to be the product of $M$ with the euclidean interval $(0, L)$ :

$$
\mathcal{G}:=(0, L) \times M
$$

with the Riemannian product metric on $\mathcal{G}$. When $M$ equals the unit circle $S^{1}$, we obtain the standard cylinder of length $L$ and radius 1 . By convention, in this section only we also allow $M$ to be empty (with $N=0$ ), in which case we identify $\mathcal{G}$ with the interval $(0, L)$. Readers interested in these two special cases can simplify the following remarks considerably.

Write $d V$ for the volume element on $M$ induced by the metric $g$, with $V(M)$ denoting the total volume of $M_{g}$. Thus we have a product measure element $d x d V(\theta)$ on $\mathcal{G}$.

Let $w \in \mathcal{L}^{\infty}(\mathcal{G})$ with $w>0$ a.e. Consider the following eigenvalue problem on $\mathcal{G}$ with mixed boundary conditions:

$$
\begin{aligned}
-\Delta \psi & =\lambda w \psi \quad \text { for }(x, \theta) \in(0, L) \times M, \\
\frac{\partial \psi}{\partial x}(0, \theta) & =\psi(L, \theta)=0 \quad \text { for all } \theta \in M .
\end{aligned}
$$

Here $\Delta=\partial^{2} / \partial x^{2}+\Delta_{g}$, where $\Delta_{g}$ is the Laplace-Beltrami operator on $M_{g}$. (When $M=S^{1}$, we have $\Delta_{g}=\partial^{2} / \partial \theta^{2}$.) We also write $\nabla=\partial / \partial x+\nabla_{g}$.

We claim that the operator $-w^{-1} \Delta$ on $\mathcal{G}$ is positive and has a discrete spectrum $\left\{\lambda_{j}(w)\right\}$, with $0<\lambda_{1}(w)<\lambda_{2}(w) \leq \lambda_{3}(w) \leq \cdots \rightarrow \infty$. Furthermore, the eigenvalues are given by Poincaré's minimax principle in terms of the Rayleigh quotient:

$$
\lambda_{j}(w)=\min _{L_{j}} \max _{\psi \in L_{j} \backslash\{0\}} \frac{\int_{\mathcal{G}}|\nabla \psi|^{2} d V(\theta) d x}{\int_{\mathcal{G}} \psi^{2} w d V(\theta) d x},
$$


where $L_{j}$ ranges over all $j$-dimensional subspaces of the trial space $H_{m i x}^{1}(\mathcal{G})$, with

$$
\begin{aligned}
H_{\text {mix }}^{1}(\mathcal{G}):= & \text { the closure in } H^{1}(\mathcal{G}) \text { of } \\
& \left\{\psi \in H^{1}(\mathcal{G}) \cap C^{\infty}(\mathcal{G}): \psi(x, \theta)=0 \text { for all } \theta, \text { when } x \text { is near } L\right\} .
\end{aligned}
$$

Here $H^{1}(\mathcal{G})$ denotes the usual Sobolev space on the generalized cylinder $\mathcal{G}$, known also as $W^{1,2}(\mathcal{G})$. Note that our trial space $H_{\text {mix }}^{1}(\mathcal{G})$ does not depend on $w$. In (5.1) and elsewhere in the paper we use the notations

$$
\begin{array}{ccc}
|\nabla \psi|^{2} & \text { for } & (\partial \psi / \partial x)^{2}+g\left(\nabla_{g} \psi, \nabla_{g} \psi\right), \\
\nabla \phi \cdot \nabla \psi & \text { for } & (\partial \phi / \partial x)(\partial \psi / \partial x)+g\left(\nabla_{g} \phi, \nabla_{g} \psi\right) .
\end{array}
$$

We claim in addition that the eigenfunctions $\psi_{j} \in H_{m i x}^{1}(\mathcal{G})$ are continuous and satisfy $-\Delta \psi_{j}=\lambda_{j}(w) w \psi_{j}$ weakly in $H_{m i x}^{1}(\mathcal{G})$, and that the first eigenfunction $\psi_{1}$ is unique up to constant factors and is never zero.

For the preceding facts, argue as in [5, pp. 53-61,71] and [12, pp. 212214]. Note that the boundedness and positivity a.e. of $w$ ensures

$$
\int_{\mathcal{G}} \psi^{2} w d V(\theta) d x
$$

is finite and positive when $\psi \in \mathcal{L}^{2}(\mathcal{G}), \psi \not \equiv 0$. The connectedness of $M$ ensures that $\lambda_{1}<\lambda_{2}$. We will neither need nor use this next remark, but it is interesting that $\psi_{j}$ is continuous on $(0, L] \times M$ with $\psi_{j}(L, \theta)=0$ for all $\theta$, which is the classical Dirichlet boundary condition, and if $\psi_{j}$ is $C^{2}$-smooth up to the boundary at $x=0$ then $\left(\partial \psi_{j} / \partial x\right)(0, \theta)=0$ for all $\theta$, which is the classical Neumann boundary condition.

Many times in this paper we use the following variational characterization of the sum of reciprocal eigenvalues. For its proof by induction from the minimax principle (5.1) and for a discussion of its history, see [2, pp. 99-100]. The characterization says that for each $m \geq 1$ :

$$
\sum_{j=1}^{m} \frac{1}{\lambda_{j}(w)}=\sup _{\left\{\psi_{1}, \ldots, \psi_{m}\right\}} \sum_{j=1}^{m} \int_{0}^{L} \int_{M} \psi_{j}^{2} w d V(\theta) d x
$$

where $\left\{\psi_{1}, \ldots, \psi_{m}\right\}$ is required to be a collection of $m$ linearly independent functions in $H_{m i x}^{1}(\mathcal{G})$ with orthonormal gradients, $\int_{0}^{L} \int_{M} \nabla \psi_{i}$. $\nabla \psi_{j} d V(\theta) d x=\delta_{i j}$.

Finally, we will need a lower bound of Weyl type: for some $\alpha \in(0,1)$ that depends on $w$,

$$
\lambda_{j}(w) \geq \alpha j^{2 /(1+N)} \quad \text { for all } j \geq 1,
$$


and this can be seen as follows. By the minimax principle above, $\lambda_{j}(w) \geq$ $\|w\|_{\infty}^{-1} \lambda_{j}(1)$, and $\lambda_{j}(1) \sim$ (const.) $j^{2 /(1+N)}$ by Weyl's law [10, p. 9] for the eigenvalues of $\Delta$ on the Riemannian manifold $\mathcal{G}$ of dimension $1+N$. Since also $\lambda_{1}(w)>0$, the lower bound (5.3) follows. The argument is even easier for the interval $(0, L)$, since there we know $\lambda_{j}(1)=(2 j-1)^{2}(\pi / 2 L)^{2}$.

Note that since $\lambda_{j}(w) \geq \alpha j^{2 /(1+N)}$, the zeta function

$$
\sum_{j=1}^{\infty} \frac{1}{\lambda_{j}(w)^{p}}
$$

of the operator $w^{-1} \Delta$ on $\mathcal{G}$ converges for $p>(1+N) / 2$.

\section{Purely Dirichlet boundary conditions.}

Redefine $\mathcal{G}:=(-L, L) \times M$. Except for some obvious changes, everything described in the previous subsection remains true for the eigenvalue problem on $\mathcal{G}$ with purely Dirichlet boundary conditions $\psi( \pm L, \theta)=0$ for all $\theta \in M$, provided the space $H_{m i x}^{1}(\mathcal{G})$ is replaced everywhere with the Sobolev space $H_{0}^{1}(\mathcal{G})$.

\section{Two trigonometric lemmas.}

The following lemma about sine functions of odd multiples of $x$ helps in proving Theorems 2,4 and 7 .

Lemma 15. Let $m \geq 2$ and suppose $\left\{c_{j}\right\}_{j=1}^{m}$ is a decreasing sequence of positive numbers, with $c_{1}>c_{2}$. Then

$$
\sum_{j=1}^{m} c_{j} \sin (2 j-1) x>0 \quad \text { for all } x \in(0, \pi) .
$$

To prove this lemma, first notice that for each fixed $k \geq 1$,

$$
\begin{aligned}
\sin x \sum_{j=1}^{k} \sin (2 j-1) x & =\frac{1}{2} \sum_{j=1}^{k}[\cos (2 j-2) x-\cos (2 j x)] \\
& =\frac{1}{2}[1-\cos (2 k x)]=\sin ^{2}(k x)
\end{aligned}
$$

and so

$$
\sum_{j=1}^{k} \sin (2 j-1) x=\frac{\sin ^{2}(k x)}{\sin x} .
$$


Next, write $c_{m+1}:=0$ and observe that

$$
\sum_{j=1}^{m} c_{j} \sin (2 j-1) x=\sum_{k=1}^{m}\left(c_{k}-c_{k+1}\right) \sum_{j=1}^{k} \sin (2 j-1) x .
$$

This last quantity is nonnegative for all $x \in(0, \pi)$ by the identity (6.1), since $c_{k} \geq c_{k+1}$, and is actually positive because the $k=1$ term is positive: $\left(c_{1}-c_{2}\right) \sin x>0$.

The next lemma, about sine functions of multiples of $x$, helps to prove Theorem 13.

Lemma 16. Let $m \geq 1$ and suppose $\left\{c_{j}\right\}_{j=1}^{m}$ is a decreasing sequence of positive numbers. Then

$$
\sum_{j=1}^{m} \frac{c_{j}}{j} \sin j x>0 \quad \text { for all } x \in(0, \pi) .
$$

To prove the lemma, write $c_{m+1}:=0$ and notice that

$$
\sum_{j=1}^{m} \frac{c_{j}}{j} \sin j x=\sum_{k=1}^{m}\left(c_{k}-c_{k+1}\right) \sum_{j=1}^{k} \frac{\sin j x}{j} .
$$

This last quantity is nonnegative for all $x \in(0, \pi)$ because $c_{k} \geq c_{k+1}$ by assumption and $\sum_{j=1}^{k}(\sin j x) / j>0$ by [29, p. 62], and the quantity is actually positive because $c_{k}>c_{k+1}$ for some $k$ (using that $c_{1}>0=c_{m+1}$ ).

\section{Proof of Theorem 1.}

Let $\psi_{1}$ be a first eigenfunction for $v^{-1} d^{2} / d x^{2}$ on $(0, L)$, so that $-\psi_{1}^{\prime \prime}=$ $\lambda_{1}(v) v \psi_{1}$ weakly in $H_{m i x}^{1}(0, L)$. We may assume $\psi_{1}$ is positive because, as we remarked in Section $5, \psi_{1}$ is continuous and never zero. To prove the theorem, simply adapt the proof of Theorem 8 in Section 13; start at (13.1), remove all occurrences of $\int_{M}$ and $d V(\theta)$, and write:

$$
\begin{array}{lll}
d / d x & \text { instead of } & \nabla, \\
d^{2} / d x^{2} & \text { instead of } & \Delta, \\
w(\cdot) & \text { instead of } & w(\cdot, \theta) \\
(2.1) & \text { instead of } & (2.15)
\end{array}
$$




\section{Proof of Theorem 2 and Corollary 3.}

We first prove the theorem, then at the end we deduce the corollary.

Assume for a moment that we know the result when $\Phi=$ id., namely

$$
\sum_{j=1}^{m} \frac{1}{\lambda_{j}(w)} \geq \sum_{j=1}^{m} \frac{1}{\lambda_{j}(1)}
$$

for each $m=1,2,3, \ldots,+\infty$, with strict inequality for each $m$ unless $w=1$ a.e. Then extend $\Phi$ to be convex and increasing from $\mathbf{R}$ to $\mathbf{R}$ by putting $\Phi(a)=0$ for $a<0$. The conclusion (2.4) then follows immediately from (8.1) and the majorization technique of Hardy, Littlewood and Pólya [18, Prop. 10]. For the equality statements of Theorem 2, suppose that equality holds in (2.4). Note that both sides of (2.4) are finite-valued, since $\lambda_{j} \geq \alpha j^{2}$ by (2.2) and since $\Phi(a) \leq a \Phi(1)$ for all $a \in(0,1)$ by convexity of $\Phi$. Also, $\Phi$ is non-constant on $\left(-\infty, \lambda_{1}(w)^{-1}\right.$ ] because $\Phi\left(\lambda_{1}(w)^{-1}\right)>0=\Phi(0)$ by hypothesis. Further, we can assume $\Phi$ is not linear on $\left[0, \lambda_{1}(w)^{-1}\right]$, because if it were linear we could simply refer to the equality statement of (8.1). Thus case (ii) of [18, Prop. 10] applies (with $\beta=0$ ) and gives that equality holds in (8.1) for some $m$, and so $w=1$ a.e.

Thus we have only to prove (8.1) and its equality statements. Fix a positive integer $m$. Recall from (5.2) that

$$
\sum_{j=1}^{m} \frac{1}{\lambda_{j}(w)}=\sup _{\left\{\psi_{1}, \ldots, \psi_{m}\right\}} \sum_{j=1}^{m} \int_{0}^{L} \psi_{j}^{2} w d x
$$

where $\left\{\psi_{1}, \ldots, \psi_{m}\right\}$ is required to be a collection of $m$ linearly independent functions in $H_{m i x}^{1}(0, L)$, with $\int_{0}^{L} \psi_{i}^{\prime} \psi_{j}^{\prime} d x=\delta_{i j}$. Take $\psi_{1}, \ldots, \psi_{m} \in$ $H_{m i x}^{1}(0, L)$ to be linearly independent smooth eigenfunctions of $d^{2} / d x^{2}$ on $[0, L]$ that satisfy $-\psi_{j}^{\prime \prime}=\lambda_{j}(1) \psi_{j}$ and

$$
\lambda_{j}(1)=\frac{\int_{0}^{L}\left|\psi_{j}^{\prime}\right|^{2} d x}{\int_{0}^{L} \psi_{j}^{2} d x} \quad \text { and } \quad \int_{0}^{L} \psi_{i}^{\prime} \psi_{j}^{\prime} d x=\delta_{i j}
$$

so that

$$
\sum_{j=1}^{m} \frac{1}{\lambda_{j}(1)}=\sum_{j=1}^{m} \int_{0}^{L} \psi_{j}^{2} d x
$$


We explicitly construct these (trigonometric) eigenfunctions $\psi_{j}$ below. By using $\psi_{1}, \ldots, \psi_{m}$ as trial functions in the characterization (8.2) we get a lower bound on $\sum_{j=1}^{m} \lambda_{j}(w)^{-1}$, with

$$
\begin{aligned}
\sum_{j=1}^{m} \frac{1}{\lambda_{j}(w)}-\sum_{j=1}^{m} \frac{1}{\lambda_{j}(1)} & \geq \sum_{j=1}^{m} \int_{0}^{L} \psi_{j}^{2}[w-1] d x \\
& =\int_{0}^{L} \sum_{j=1}^{m} \psi_{j}(s)^{2} \frac{d}{d s}\left\{\int_{0}^{s} w(x) d x-s\right\} d s \\
& =-\int_{0}^{L}\left\{\int_{0}^{s} w(x) d x-s\right\} \frac{d}{d s} \sum_{j=1}^{m} \psi_{j}(s)^{2} d s
\end{aligned}
$$

by parts, since $\psi_{j}(L)=0$. Next, recall the hypothesis (2.3), which says that $\int_{0}^{s} w(x) d x \geq s$ for all $s$. Thus it suffices to show that

$$
\frac{d}{d s} \sum_{j=1}^{m} \psi_{j}(s)^{2}<0 \quad \text { for all } s \in(0, L),
$$

because then

$$
\sum_{j=1}^{m} \frac{1}{\lambda_{j}(w)} \geq \sum_{j=1}^{m} \frac{1}{\lambda_{j}(1)}
$$

with strict inequality unless $\int_{0}^{s} w(x) d x=s$ for all $s$ (i.e., unless $w(x)=1$ for almost all $x$ ), and this gives (8.1) (except for the strict inequality statement for $m=+\infty$, which we handle later).

Our immediate goal, then, is to establish (8.5). For this we use the following explicit formulas for the eigenfunctions $\psi_{j}$ and eigenvalues $\lambda_{j}(1)$ :

$$
\psi_{j}(x):=\frac{\sqrt{8 L}}{(2 j-1) \pi} \cos \left(\frac{(2 j-1) \pi x}{2 L}\right) \quad \text { and } \quad \lambda_{j}(1)=\left(\frac{(2 j-1) \pi}{2 L}\right)^{2} .
$$

Clearly the $\psi_{j}$ are indeed eigenfunctions of $d^{2} / d x^{2}$ on the interval $(0, L)$, with eigenvalues $\lambda_{j}(1)$, and they satisfy the boundary conditions $\psi_{j}^{\prime}(0)=$ $\psi_{j}(L)=0$ and also the normalizing conditions (8.3). By substituting the $\psi_{j}$ into (8.5) and differentiating, we see that it is enough to show

$$
\sum_{j=1}^{m} \frac{\sin (2 j-1) x}{(2 j-1)}>0 \quad \text { for all } x \in(0, \pi) .
$$

Since this follows from Lemma 15 with $c_{j}:=(2 j-1)^{-1}$, we have proved (8.5). 
To complete the proof of Theorem 2 it remains only to prove the strict inequality statement of (8.1) when $m=+\infty$. Along the way we will give a direct proof of (8.1) itself for $m=+\infty$. Start by letting $m \rightarrow \infty$ in (8.4), which yields

$$
\sum_{j=1}^{\infty} \frac{1}{\lambda_{j}(w)}-\sum_{j=1}^{\infty} \frac{1}{\lambda_{j}(1)} \geq \int_{0}^{L} \sum_{j=1}^{\infty} \psi_{j}^{2}[w-1] d x .
$$

Using the definition of $\psi_{j}$ and the double angle formula for $\cos ^{2}$ gives that

$$
\begin{aligned}
\sum_{j=1}^{\infty} \psi_{j}(x)^{2} & =\frac{8 L}{\pi^{2}} \sum_{j=1}^{\infty} \frac{1}{(2 j-1)^{2}} \cos ^{2}\left(\frac{(2 j-1) \pi x}{2 L}\right) \\
& =\frac{4 L}{\pi^{2}} \sum_{k \text { odd }} \frac{1}{k^{2}}+\frac{4 L}{\pi^{2}} \sum_{k \text { odd }} \frac{\cos (k \pi x / L)}{k^{2}} \\
& =\frac{L}{2}+\frac{4 L}{\pi^{2}} \sum_{k \text { odd }} \frac{\cos (k \pi x / L)}{k^{2}} .
\end{aligned}
$$

This last expression is the Fourier cosine series of the even function $L-|x|$ for $x \in[-L, L]$, and so

$$
\sum_{j=1}^{\infty} \psi_{j}(x)^{2}=L-x \quad \text { for all } x \in(0, L) .
$$

Hence by (8.6),

$$
\begin{aligned}
\sum_{j=1}^{\infty} \frac{1}{\lambda_{j}(w)}-\sum_{j=1}^{\infty} \frac{1}{\lambda_{j}(1)} & \geq \int_{0}^{L}(L-x)[w(x)-1] d x \\
& =\int_{0}^{L}\left\{\int_{0}^{s} w(x) d x-s\right\} d s
\end{aligned}
$$

by parts. Now (8.1) with $m=+\infty$ follows from the hypothesis that $\int_{0}^{s} w(x) d x \geq s$ for all $s$, and if equality holds in (8.1) for $m=+\infty$ then (8.7) implies $\int_{0}^{s} w(x) d x=s$ for all $s$, or $w(x)=1$ for almost all $x$, completing the proof.

The inequality (8.7) is actually an equality for all $w$, for the reasons given at the end of $\S 5$ of [17]. This gives us a physical interpretation of the $\operatorname{sum} \sum_{j=1}^{\infty} \lambda_{j}(w)^{-1}$ of the inverse square frequencies of our inhomogeneous 
string:

$$
\begin{aligned}
\sum_{j=1}^{\infty} \frac{1}{\lambda_{j}(w)} & =\int_{0}^{L}(L-x) w(x) d x \\
& =(\text { the gravitational moment about the fixed endpoint at } x=L) .
\end{aligned}
$$

See [28, Ch. V,VII], [27], [11], [17, §5], and the references therein, for more on Green functions and sums of infinitely many reciprocal eigenvalues, in one dimension.

Finally, we prove Corollary 3. Fix $p \geq 1$ and let $\Phi(a):=a^{p}$ for $a \geq 0$, so that $\Phi$ is convex and strictly increasing. The inequality in the Corollary follows immediately from Theorem 2 , and because $\lambda_{j}(1)=[(2 j-1) \pi / 2 L]^{2}$, the equality in the Corollary is easily established:

$$
\sum_{j=1}^{\infty} \frac{1}{\lambda_{j}(1)^{p}}=\left(\frac{2 L}{\pi}\right)^{2 p} \sum_{k \text { odd }} \frac{1}{k^{2 p}}=\left(\frac{L}{\pi}\right)^{2 p}\left(2^{2 p}-1\right) \zeta(2 p) .
$$

\section{Proof of Theorem 4 .}

Our initial goal is to prove the Theorem without the $\Phi$ 's, that is,

$$
\sum_{j=1}^{m} \frac{1}{\lambda_{j}(w)} \geq \sum_{j=1}^{m} \frac{1}{\lambda_{j}(1)}
$$

for each $m=1,2,3, \ldots$, with strict inequality for $m \geq 2$ unless

$$
\int_{0}^{2 \pi} w(x, \theta) d \theta=2 \pi
$$

for almost all $x \in(0, L)$, and strict inequality for $m=1$ unless $w=1$ a.e. Assume (9.1) for now, and extend $\Phi$ to be convex and increasing from $\mathbf{R}$ to $\mathbf{R}$ by putting $\Phi(a)=0$ for $a<0$. The conclusion (2.6) then follows immediately from (9.1) and the majorization technique of Hardy, Littlewood and Pólya [18, Prop. 10]. For the equality statements of Theorem 4, suppose that equality holds in (2.6). Note that both sides of (2.6) are finite-valued, since $\lambda_{j} \geq \alpha j$ by (5.3) and $\int_{1}^{n} \Phi(1 / a) d a$ is finite by hypothesis. Also, $\Phi$ is non-constant on $\left(-\infty, \lambda_{1}(w)^{-1}\right.$ ] because $\Phi\left(\lambda_{1}(w)^{-1}\right)>0=\Phi(0)$ by hypothesis. Further, if $n=+\infty$ then $\Phi$ is not linear on $\left[0, \lambda_{1}(w)^{-1}\right]$, because $\int_{1}^{n} \Phi(1 / a) d a$ is finite. Thus either $n<+\infty$ and case (iv) of the equality 
statement of [18, Prop. 10] applies, or else $n=+\infty$ and case (ii) of [18, Prop. 10] applies (with $\beta=0$ ). In any event, we get from [18, Prop. 10] that equality holds in (9.1) for some $m$, and so $\int_{0}^{2 \pi} w(x, \theta) d \theta=2 \pi$ for almost all $x \in(0, L)$. If in addition $\Phi(a)$ is strictly convex for $a \geq 0$, then by case (v) of the equality statement of [18, Prop. 10] we obtain that $1 / \lambda_{1}(w)=1 / \lambda_{1}(1)$. Hence equality holds in (9.1) for $m=1$, and so $w=1$ a.e., as desired.

In proving (9.1), we can assume $\lambda_{m+1}(1)>\lambda_{m}(1)$, as we now explain. For suppose that (9.1) and its equality statements are known to hold for all $m$ satisfying $\lambda_{m+1}(1)>\lambda_{m}(1)$. Note that $\lambda_{m+1}(1)>\lambda_{m}(1)$ for infinitely many $m$ values, in particular for $m=1$. Suppose $m_{1}$ and $m_{2}$ are consecutive such values and that $m_{2}=m_{1}+k$ for some $k \geq 2$, so that

$$
\lambda_{m_{1}}(1)<\lambda_{m_{1}+1}(1)=\cdots=\lambda_{m_{1}+k}(1) .
$$

We wish to show that (9.1) and its equality statements hold for all $m$ between $m_{1}$ and $m_{1}+k$. But if $m_{1}<m<m_{1}+k$ then either $\lambda_{m}(w) \geq \lambda_{m}(1)$, in which case $\lambda_{j}(w) \geq \lambda_{j}(1)$ for all $j$ between $m$ and $m_{1}+k$ inclusive and so

$$
\begin{aligned}
\sum_{j=1}^{m} \frac{1}{\lambda_{j}(w)} & =\sum_{j=1}^{m_{1}+k} \frac{1}{\lambda_{j}(w)}-\sum_{j=m+1}^{m_{1}+k} \frac{1}{\lambda_{j}(w)} \\
& \geq \sum_{j=1}^{m_{1}+k} \frac{1}{\lambda_{j}(1)}-\sum_{j=m+1}^{m_{1}+k} \frac{1}{\lambda_{j}(1)} \\
& =\sum_{j=1}^{m} \frac{1}{\lambda_{j}(1)}
\end{aligned}
$$

or else $\lambda_{m}(w)<\lambda_{m}(1)$, in which case $\lambda_{j}(w)<\lambda_{j}(1)$ for all $j$ between $m_{1}+1$ and $m$ inclusive and so

$$
\begin{aligned}
\sum_{j=1}^{m} \frac{1}{\lambda_{j}(w)} & =\sum_{j=1}^{m_{1}} \frac{1}{\lambda_{j}(w)}+\sum_{j=m_{1}+1}^{m} \frac{1}{\lambda_{j}(w)} \\
& >\sum_{j=1}^{m_{1}} \frac{1}{\lambda_{j}(1)}+\sum_{j=m_{1}+1}^{m} \frac{1}{\lambda_{j}(1)} \\
& =\sum_{j=1}^{m} \frac{1}{\lambda_{j}(1)} .
\end{aligned}
$$

Thus (9.1) and its equality statements hold for all $m$ between $m_{1}$ and $m_{1}+k$, as promised. Hence we can assume from now on that $\lambda_{m+1}(1)>\lambda_{m}(1)$. 
We begin to prove (9.1) by collecting facts about the eigenvalues and eigenfunctions of the homogeneous cylinder $\mathcal{C}$, with $w \equiv 1$. To start with, the eigenvalues $\left\{\lambda_{j}(1): j=1,2,3, \ldots\right\}$ can be computed by separation of variables to be

$$
\left\{\lambda_{k \ell}=k^{2}+(2 \ell-1)^{2}(\pi / 2 L)^{2}: k \in \mathbf{Z}, \ell \geq 1\right\},
$$

with corresponding normalized eigenfunctions

$$
\psi_{k \ell}(x, \theta):=\sqrt{2 /\left(\pi L \lambda_{k \ell}\right)} \cos [(2 \ell-1) \pi x / 2 L] \times\left\{\begin{array}{ll}
\sin k \theta, & \text { if } k>0 \\
1 / \sqrt{2}, & \text { if } k=0 \\
\cos k \theta, & \text { if } k<0
\end{array}\right\}
$$

satisfying $-\Delta \psi_{k \ell}=\lambda_{k \ell} \psi_{k \ell}$. Now, $\left\{\psi_{k \ell}\right\}$ is a linearly independent set in $H_{m i x}^{1}(\mathcal{C})$ and it satisfies the orthonormality condition

$$
\int_{\mathcal{C}} \nabla \psi_{k \ell} \cdot \nabla \psi_{k^{\prime} \ell^{\prime}} d \theta d x=\delta_{k k^{\prime}} \delta_{\ell \ell^{\prime}}
$$

Let

$$
I:=\left\{(k, \ell): \lambda_{k \ell} \leq \lambda_{m}(1)\right\} .
$$

Then $I$ contains exactly $m$ elements since $\lambda_{m}(1)<\lambda_{m+1}(1)$, and the numbers $\lambda_{k \ell}$ for $(k, \ell) \in I$ are a permutation of the eigenvalues $\lambda_{j}(1)$ for $j=1, \ldots, m$.

From (5.2) we have

$$
\sum_{j=1}^{m} \frac{1}{\lambda_{j}(w)}=\sup _{\left\{\psi_{1}, \ldots, \psi_{m}\right\}} \sum_{j=1}^{m} \int_{\mathcal{C}} \psi_{j}^{2} w d \theta d x
$$

where $\left\{\psi_{1}, \ldots, \psi_{m}\right\}$ is required to be a collection of $m$ linearly independent functions in $H_{m i x}^{1}(\mathcal{C})$, with $\int_{\mathcal{C}} \nabla \psi_{i} \cdot \nabla \psi_{j} d \theta d x=\delta_{i j}$. By using the functions $\psi_{k \ell}$ as trial functions in this variational characterization, we obtain that

$$
\begin{aligned}
\sum_{j=1}^{m} \frac{1}{\lambda_{j}(w)} \geq & \sum_{(k, \ell) \in I} \int_{\mathcal{C}}\left|\psi_{k \ell}\right|^{2} w d \theta d x \\
= & \sum_{(k, \ell) \in I} \frac{2}{\pi L \lambda_{k \ell}} \int_{0}^{L} \cos ^{2}[(2 \ell-1) \pi x / 2 L] \\
& \quad \times \int_{0}^{2 \pi}\left\{\begin{array}{ll}
\sin ^{2} k \theta, & \text { if } k>0 \\
1 / 2, & \text { if } k=0 \\
\cos ^{2} k \theta, & \text { if } k<0
\end{array}\right\} w(x, \theta) d \theta d x .
\end{aligned}
$$


Since $(k, \ell) \in I$ if and only if $(-k, \ell) \in I$, we deduce that

$$
\begin{aligned}
\sum_{j=1}^{m} \frac{1}{\lambda_{j}(w)} & \geq \sum_{(k, \ell) \in I} \frac{1}{\pi L \lambda_{k \ell}} \int_{0}^{L} \cos ^{2}[(2 \ell-1) \pi x / 2 L] \int_{0}^{2 \pi} w(x, \theta) d \theta d x \\
& =\sum_{(k, \ell) \in I} \frac{2 \ell-1}{2 L^{2} \lambda_{k \ell}} \int_{0}^{L} \sin [(2 \ell-1) \pi s / L] \int_{0}^{s} \int_{0}^{2 \pi} w(x, \theta) d \theta d x d s
\end{aligned}
$$

by parts in $x$. We show below that

$$
\sum_{(k, \ell) \in I} \frac{2 \ell-1}{\lambda_{k \ell}} \sin [(2 \ell-1) \pi s / L]>0 \quad \text { for all } s \in(0, L),
$$

and so from the hypothesis $\int_{0}^{s} \int_{0}^{2 \pi} w(x, \theta) d \theta d x \geq 2 \pi s$ in the theorem it follows that

$$
\begin{aligned}
\sum_{j=1}^{m} \frac{1}{\lambda_{j}(w)} & \geq \sum_{(k, \ell) \in I} \frac{2 \ell-1}{L^{2} \lambda_{k \ell}} \int_{0}^{L} \sin [(2 \ell-1) \pi s / L] \pi s d s \\
& =\sum_{(k, \ell) \in I} \frac{1}{\lambda_{k \ell}}=\sum_{j=1}^{m} \frac{1}{\lambda_{j}(1)},
\end{aligned}
$$

giving (9.1). If equality holds in (9.1), then by the above argument, $\int_{0}^{s} \int_{0}^{2 \pi} w(x, \theta) d \theta d x=2 \pi s$ for all $s$ and so $\int_{0}^{2 \pi} w(x, \theta) d \theta=2 \pi$ for almost all $x$. Lastly, if equality holds in (9.1) for $m=1$, then the equality statement of Theorem 8 (with $v \equiv 1, M=S^{1}$ ) gives that $w=1$ a.e.

To complete the proof of the theorem, we must establish (9.2), or equivalently,

$$
\sum_{\ell=1}^{\ell^{*}} c_{\ell} \sin (2 \ell-1) x>0 \quad \text { for all } x \in(0, \pi)
$$

where

$$
\begin{aligned}
I(\ell) & :=\{k:(k, \ell) \in I\}, \\
\ell^{*} & :=\text { the largest } \ell \geq 1 \text { such that } I(\ell) \text { is non-empty, } \\
c_{\ell} & :=\sum_{k \in I(\ell)} \frac{(2 \ell-1)(\pi / 2 L)}{k^{2}+(2 \ell-1)^{2}(\pi / 2 L)^{2}} .
\end{aligned}
$$

Now, Lemma 15 in Section 6 will imply (9.3) once we show that $\left\{c_{\ell}\right\}$ is strictly decreasing. (We can assume $\ell^{*} \geq 2$ since (9.3) is trivial when $\ell^{*}=$ 
1.) To show $c_{\ell}>c_{\ell+1}$, first write $I(\ell+1)=\{-K, \cdots, K\}$ and $I(\ell)=$ $\left\{-K^{*}, \cdots, K^{*}\right\}$, where $K \leq K^{*}$ since $I(\ell+1) \subset I(\ell)$. Then invoke the next lemma at $y_{1}=(2 \ell-1)(\pi / 2 L)$ and $y_{2}=(2(\ell+1)-1)(\pi / 2 L)$ to deduce that

$$
c_{\ell} \geq \sum_{k=-K}^{K} \frac{y_{1}}{k^{2}+y_{1}^{2}}>\sum_{k=-K}^{K} \frac{y_{2}}{k^{2}+y_{2}^{2}}=c_{\ell+1} .
$$

This completes the proof of Theorem 4 .

Lemma 17. For each fixed nonnegative integer $K$,

$$
y \mapsto \sum_{k=-K}^{K} \frac{y}{k^{2}+y^{2}}
$$

is strictly decreasing for $y>0$.

The lemma is true for $y>K$ by direct differentiation. For $0<y \leq K$ we observe that

$$
\begin{aligned}
\sum_{k=-K}^{K} \frac{y}{k^{2}+y^{2}} & =\sum_{k=-\infty}^{\infty} \frac{y}{k^{2}+y^{2}}-\sum_{|k|>K} \frac{y}{k^{2}+y^{2}} \\
& =\pi \operatorname{coth}(\pi y)-\sum_{|k|>K} \frac{y}{k^{2}+y^{2}}
\end{aligned}
$$

using a series for coth [13, p. 44], and this proves the lemma because coth is strictly decreasing and $y \mapsto \sum_{|k|>K} y /\left(k^{2}+y^{2}\right)$ is strictly increasing (for $0<y \leq K)$.

\section{Evidence for Computational Result 5.}

As usual, we begin by reducing to the case $\Phi=\mathrm{id}$. Assume for a moment that we know

$$
\sum_{j=1}^{m} \frac{1}{\lambda_{j}(w)} \geq \sum_{j=1}^{m} \frac{1}{\lambda_{j}(1)}
$$

for each $m$ with $1 \leq m \leq 53055$, with strict inequality when $m \geq 2$ unless

$$
\int_{0}^{2 \pi} \int_{0}^{2 \pi} w\left(x, \theta_{1}, \theta_{2}\right) d \theta_{1} d \theta_{2}=(2 \pi)^{2} \quad \text { for almost all } x \in(0, \pi / 2),
$$


and strict inequality when $m=1$ unless $w=1$ a.e. Now extend $\Phi$ to be convex and increasing from $\mathbf{R}$ to $\mathbf{R}$ by putting $\Phi(a)=\Phi(0)$ for $a<0$. The conclusion (2.8) then follows immediately from (10.1) and the majorization technique of Hardy, Littlewood and Pólya [18, Prop. 10], by setting $a_{j}:=$ $\lambda_{j}(w)^{-1}$ and $b_{j}:=\lambda_{j}(1)^{-1}$ for $1 \leq j \leq 53055$, and setting $a_{j}:=0$ and $b_{j}:=0$ for $j>53055$. For the equality statement of (2.8), suppose that equality holds in (2.8) and note that both sides of (2.8) are finite-valued since $n$ is finite. Also, $\Phi$ is non-constant on $\left(-\infty, \lambda_{1}(w)^{-1}\right.$ ] since $\Phi\left(\lambda_{1}(w)^{-1}\right)>\Phi(0)$ by hypothesis in the Computational Result. Thus case (iv) of the equality statement of [18, Prop. 10] applies and we get that equality holds in (10.1) for some $m$, and so $\int_{0}^{2 \pi} \int_{0}^{2 \pi} w\left(x, \theta_{1}, \theta_{2}\right) d \theta_{1} d \theta_{2}=(2 \pi)^{2}$ for almost all $x \in$ $(0, \pi / 2)$. If in addition $\Phi(a)$ is strictly convex for $a \geq 0$, then by case (v) of the equality statement of [18, Prop. 10] we obtain that $\lambda_{1}(w)^{-1}=\lambda_{1}(1)^{-1}$. Hence equality holds in (10.1) for $m=1$, and so $w=1$ a.e., as desired.

We have still to try to prove (10.1) and its equality statements. The case $m=1$ of (10.1) is a simple case of Theorem 8 (with $v \equiv 1, M=S^{1} \times S^{1}$ ), which we prove in Section 13, and so we can fix $m$ with $2 \leq m \leq 53055$ from now on.

In establishing (10.1), we can also assume $\lambda_{m+1}(1)>\lambda_{m}(1)$, by the same reasoning as we used in the proof of Theorem 4 . Note here that indeed $\lambda_{m+1}(1)>\lambda_{m}(1)$ for $m=53055$, by the computer calculations summarized in (2.10).

We begin to establish (10.1). To start with, the eigenvalues $\left\{\lambda_{j}(1): j=\right.$ $1,2,3, \ldots\}$ can be computed by separation of variables to be

$$
\left\{\lambda_{k_{1} k_{2} \ell}=k_{1}^{2}+k_{2}^{2}+(2 \ell-1)^{2}: k_{1}, k_{2} \in \mathbf{Z}, \ell \geq 1\right\},
$$

with corresponding normalized eigenfunctions

$$
\begin{aligned}
\psi_{k_{1} k_{2} \ell}\left(x, \theta_{1}, \theta_{2}\right):=\frac{2}{\sqrt{\pi^{3} \lambda_{k_{1} k_{2} \ell}}} & \cos [(2 \ell-1) x]\left\{\begin{array}{ll}
\sin k_{1} \theta_{1}, & \text { if } k_{1}>0 \\
1 / \sqrt{2}, & \text { if } k_{1}=0 \\
\cos k_{1} \theta_{1}, & \text { if } k_{1}<0
\end{array}\right\} \\
& \times\left\{\begin{array}{ll}
\sin k_{2} \theta_{2}, & \text { if } k_{2}>0 \\
1 / \sqrt{2}, & \text { if } k_{2}=0 \\
\cos k_{2} \theta_{2}, & \text { if } k_{2}<0
\end{array}\right\}
\end{aligned}
$$

satisfying $-\Delta \psi_{k_{1} k_{2} \ell}=\lambda_{k_{1} k_{2} \ell} \psi_{k_{1} k_{2} \ell}$. Now, $\left\{\psi_{k_{1} k_{2} \ell}\right\}$ is a linearly independent set in $H_{m i x}^{1}(\mathcal{D})$ and it satisfies the orthonormality condition

$$
\int_{\mathcal{D}} \nabla \psi_{k_{1} k_{2} \ell} \cdot \nabla \psi_{k_{1}^{\prime} k_{2}^{\prime} \ell^{\prime}} d \theta_{1} d \theta_{2} d x=\delta_{k_{1} k_{1}^{\prime}} \delta_{k_{2} k_{2}^{\prime}} \delta_{\ell \ell^{\prime}}
$$


Let

$$
I:=\left\{\left(k_{1}, k_{2}, \ell\right): \lambda_{k_{1} k_{2} \ell} \leq \lambda_{m}(1)\right\} .
$$

Then $I$ contains exactly $m$ elements, since $\lambda_{m+1}(1)>\lambda_{m}(1)$. The numbers $\lambda_{k_{1} k_{2} \ell}$ for $\left(k_{1}, k_{2}, \ell\right) \in I$ are a permutation of the eigenvalues $\lambda_{j}(1)$ for $j=$ $1, \ldots, m$.

From (5.2) we know that

$$
\sum_{j=1}^{m} \frac{1}{\lambda_{j}(w)}=\sup _{\left\{\psi_{1}, \ldots, \psi_{m}\right\}} \sum_{j=1}^{m} \int_{\mathcal{D}} \psi_{j}^{2} w d \theta_{1} d \theta_{2} d x,
$$

where $\left\{\psi_{1}, \ldots, \psi_{m}\right\}$ is required to be a collection of $m$ linearly independent functions in $H_{m i x}^{1}(\mathcal{D})$, with $\int_{\mathcal{D}} \nabla \psi_{i} \cdot \nabla \psi_{j} d \theta_{1} d \theta_{2} d x=\delta_{i j}$. By using the functions $\psi_{k_{1} k_{2} \ell}$ as trial functions in this variational characterization, we obtain that

$$
\begin{aligned}
\sum_{j=1}^{m} \frac{1}{\lambda_{j}(w)} \geq & \sum_{\left(k_{1}, k_{2}, \ell\right) \in I} \int_{\mathcal{D}}\left|\psi_{k_{1} k_{2} \ell}\right|^{2} w d \theta_{1} d \theta_{2} d x \\
= & \sum_{\left(k_{1}, k_{2}, \ell\right) \in I} \frac{4}{\pi^{3} \lambda_{k_{1} k_{2} \ell}} \int_{0}^{\pi / 2} \cos ^{2}[(2 \ell-1) x] \int_{0}^{2 \pi} \int_{0}^{2 \pi} \\
& \times\left\{\begin{array}{ll}
\sin ^{2} k_{1} \theta_{1}, & \text { if } k_{1}>0 \\
1 / 2, & \text { if } k_{1}=0 \\
\cos ^{2} k_{1} \theta_{1}, & \text { if } k_{1}<0
\end{array}\right\} \\
& \times\left\{\begin{array}{ll}
\sin ^{2} k_{2} \theta_{2}, & \text { if } k_{2}>0 \\
1 / 2, & \text { if } k_{2}=0 \\
\cos ^{2} k_{2} \theta_{2}, & \text { if } k_{2}<0
\end{array}\right\} w\left(x, \theta_{1}, \theta_{2}\right) d \theta_{1} d \theta_{2} d x .
\end{aligned}
$$

Since $\left(k_{1}, k_{2}, \ell\right) \in I$ if and only if $\left(-k_{1}, k_{2}, \ell\right) \in I$, and $\left(k_{1}, k_{2}, \ell\right) \in I$ if and only if $\left(k_{1},-k_{2}, \ell\right) \in I$,

$$
\begin{aligned}
\sum_{j=1}^{m} \frac{1}{\lambda_{j}(w)} \geq \sum_{\left(k_{1}, k_{2}, \ell\right) \in I} \frac{1}{\pi^{3} \lambda_{k_{1} k_{2} \ell}} \int_{0}^{\pi / 2} \cos ^{2}[(2 \ell-1) x] \\
\quad \times \int_{0}^{2 \pi} \int_{0}^{2 \pi} w\left(x, \theta_{1}, \theta_{2}\right) d \theta_{1} d \theta_{2} d x \\
=\sum_{\left(k_{1}, k_{2}, \ell\right) \in I} \frac{2 \ell-1}{\pi^{3} \lambda_{k_{1} k_{2} \ell}} \int_{0}^{\pi / 2} \sin [2(2 \ell-1) s] \\
\quad \times \int_{0}^{s} \int_{0}^{2 \pi} \int_{0}^{2 \pi} w\left(x, \theta_{1}, \theta_{2}\right) d \theta_{1} d \theta_{2} d x d s
\end{aligned}
$$


by parts in $x$. We show below (numerically) that

$$
\sum_{\left(k_{1}, k_{2}, \ell\right) \in I} \frac{2 \ell-1}{\lambda_{k_{1} k_{2} \ell}} \sin [2(2 \ell-1) s]>0 \quad \text { for all } s \in(0, \pi / 2),
$$

and so from the hypothesis $\int_{0}^{s} \int_{0}^{2 \pi} \int_{0}^{2 \pi} w\left(x, \theta_{1}, \theta_{2}\right) d \theta_{1} d \theta_{2} d x \geq(2 \pi)^{2} s$ it follows that

$$
\begin{aligned}
\sum_{j=1}^{m} \frac{1}{\lambda_{j}(w)} & \geq \sum_{\left(k_{1}, k_{2}, \ell\right) \in I} \frac{4(2 \ell-1)}{\pi \lambda_{k_{1} k_{2} \ell}} \int_{0}^{\pi / 2} \sin [2(2 \ell-1) s] s d s \\
& =\sum_{\left(k_{1}, k_{2}, \ell\right) \in I} \frac{1}{\lambda_{k_{1} k_{2} \ell}}=\sum_{j=1}^{m} \frac{1}{\lambda_{j}(1)},
\end{aligned}
$$

giving (10.1). If equality holds in (10.1), then by the above argument,

$$
\int_{0}^{s} \int_{0}^{2 \pi} \int_{0}^{2 \pi} w\left(x, \theta_{1}, \theta_{2}\right) d \theta_{1} d \theta_{2} d x=(2 \pi)^{2} s
$$

for all $s$ and so $\int_{0}^{2 \pi} \int_{0}^{2 \pi} w\left(x, \theta_{1}, \theta_{2}\right) d \theta_{1} \theta_{2}=(2 \pi)^{2}$ for almost all $x$, as desired.

The "proof" of the Computational Result would be complete if we could rigorously prove (10.2), or equivalently,

$$
\sum_{\ell=1}^{\ell^{*}} d_{\ell} \sin [(2 \ell-1) x]>0 \quad \text { for all } x \in(0, \pi),
$$

where (with $A=\lambda_{m}(1)$ )

$$
\begin{aligned}
I(\ell) & :=\left\{\left(k_{1}, k_{2}\right): k_{1}^{2}+k_{2}^{2}+(2 \ell-1)^{2} \leq A\right\}, \\
\ell^{*} & :=\text { the largest } \ell \geq 1 \text { such that } I(\ell) \text { is non-empty, } \\
d_{\ell} & :=\sum_{\left(k_{1}, k_{2}\right) \in I(\ell)} \frac{(2 \ell-1)}{k_{1}^{2}+k_{2}^{2}+(2 \ell-1)^{2}} .
\end{aligned}
$$

Since we want this to hold for all $m \leq 53055$ and since, by (2.10), $\lambda_{m}(1)$ is an integer less than or equal to 1370 , it suffices to establish (10.3) for all positive integers $A \leq 1370$. Now, for $A \leq 8$ (or $m \leq 21$ ), (10.3) is obviously true because $\ell^{*}=1$. And for $8<A \leq 24$ (or $21<m \leq 114$ ) one can check by hand that $\ell^{*}=2$ and that $d_{1}>d_{2}$ for each $A$, so that (10.3) holds by Lemma 15. For $A=25$ (or $m=119$ ) we get $\ell^{*}=3$ and $d_{1}>d_{2}>d_{3}$, and so again (10.3) holds by Lemma 15. However, this method breaks down for 
$A=26$ (or $m=143$ ) because one then has $\ell^{*}=3$ and $d_{2}>d_{1}>d_{3}$, so that Lemma 15 cannot be used. In fact, it is not clear to me how to prove (10.3) rigorously for $26 \leq A \leq 1370$ (particularly for large $A$ ), but I used a double precision Fortran program to check numerically that the lefthand side of (10.3) is indeed positive for each $A \leq 1370$ at each of the million $x$-values: $x=\pi i / 10^{6}, 0<i<10^{6}$. The program tests at each $x$-value to see whether the lefthand side of (10.3) is less than $10^{-4}$; this tolerance of $10^{-4}$ should allow plenty of room for round-off errors to be detected.

The above method of proof for Computational Result 5 breaks down for $m=53199$, because the inequality (10.3) fails for $A=1371$. I cannot show this by hand, but a simple computer program can be used to show that when $A=1371$, the lefthand side of (10.3) is negative at $x=0.446 \pi$, equalling approximately -0.02 . Equivalently, the lefthand side of (10.2) is negative at $s=0.223 \pi$.

It does not follow that Computational Result 5 itself fails for $m=53199$, but it fails nonetheless. We omit the proof of this failure (i.e., of (2.9)) because it follows the lines of [17, Theorem 3].

\section{Proof of Theorem 6 .}

Again we commence by reducing to the case $\Phi=\mathrm{id}$. Assume to begin with that we know

$$
\sum_{j=1}^{m} \frac{1}{\lambda_{j}(w)} \geq \sum_{j=1}^{m} \frac{1}{\lambda_{j}(v)}
$$

for each $m=1,2,3, \ldots$, with strict inequality when $m \geq 2$ unless $\int_{M} w(x, \theta) d V(\theta)=V(M) v(x)$ for almost all $x \in(0, L)$ and strict inequality when $m=1$ unless $w=v$ a.e. Then (2.12) and its equality statements can be proved by adapting the majorization argument in the first paragraph of Section 9 (but of course, using that $\int_{1}^{n} \Phi\left(1 / a^{2 /(1+N)}\right) d a$ is finite rather than that $\int_{1}^{n} \Phi(1 / a) d a$ is finite).

Thus we have only to prove (11.1) and its equality statements. Fix $m \geq 1$. Take $\psi_{1}, \ldots, \psi_{m} \in H_{m i x}^{1}((0, L) \times M)$ to be linearly independent eigenfunctions of $v^{-1} \Delta$ on $(0, L) \times M$ that satisfy

$$
\lambda_{j}(v)=\frac{\int_{0}^{L} \int_{M}\left|\nabla \psi_{j}\right|^{2} d V(\theta) d x}{\int_{0}^{L} \int_{M} \psi_{j}^{2} v d V(\theta) d x} \quad \text { and } \quad \int_{0}^{L} \int_{M} \nabla \psi_{i} \cdot \nabla \psi_{j} d V(\theta) d x=\delta_{i j},
$$


so that

$$
\sum_{j=1}^{m} \frac{1}{\lambda_{j}(v)}=\sum_{j=1}^{m} \int_{0}^{L} \int_{M} \psi_{j}^{2} v d V(\theta) d x
$$

By using $\psi_{1}, \ldots, \psi_{m}$ as trial functions in the characterization (5.2) we get a lower bound on $\sum_{j=1}^{m} \lambda_{j}(w)^{-1}$, so that

$$
\begin{aligned}
\sum_{j=1}^{m} \frac{1}{\lambda_{j}(w)}-\sum_{j=1}^{m} \frac{1}{\lambda_{j}(v)} & \geq \sum_{j=1}^{m} \int_{0}^{L} \int_{M} \psi_{j}^{2}[w-v] d V(\theta) d x \\
& =\int_{0}^{L} \int_{M} \sum_{j=1}^{m} \psi_{j}(x, \theta)^{2}[w(x, \theta)-v(x)] d V(\theta) d x
\end{aligned}
$$

Next, because $v(x)$ is independent of $\theta \in M$, for each isometry $\tau$ of $M_{g}$ we have that the functions $\psi_{1}(x, \tau(\theta)), \ldots, \psi_{m}(x, \tau(\theta))$ are eigenfunctions of $v^{-1} \Delta$ on $M$ that satisfy (11.2). Hence by the same argument as above,

$$
\sum_{j=1}^{m} \frac{1}{\lambda_{j}(w)}-\sum_{j=1}^{m} \frac{1}{\lambda_{j}(v)} \geq \int_{0}^{L} \int_{M} \sum_{j=1}^{m} \psi_{j}(x, \tau(\theta))^{2}[w(x, \theta)-v(x)] d V(\theta) d x
$$

The isometry group $G$ of $M_{g}$ is a compact Lie group with respect to the compact-open topology (see [15, Theorem VI.3.4]), and $G$ is not 0dimensional in view of its transitivity on $M_{g}$. Hence $G$ possesses a normalized bi-invariant Haar measure $H$ (see [6, pp. 247,251]). Integrating over all isometries $\tau$ (with respect to $H$ ) gives that

$$
\begin{aligned}
\sum_{j=1}^{m} \frac{1}{\lambda_{j}(w)} & -\sum_{j=1}^{m} \frac{1}{\lambda_{j}(v)} \\
& \geq \int_{0}^{L} \int_{M} \int_{G} \sum_{j=1}^{m} \psi_{j}(x, \tau(\theta))^{2} d H(\tau)[w(x, \theta)-v(x)] d V(\theta) d x
\end{aligned}
$$

Furthermore, the right-invariance of Haar measure and the transitivity of the isometry group (that is, the homogeneity of $M$ ) imply that for some fixed $\theta_{0} \in M$,

$$
\int_{G} \sum_{j=1}^{m} \psi_{j}(x, \tau(\theta))^{2} d H(\tau)=\int_{G} \sum_{j=1}^{m} \psi_{j}\left(x, \tau\left(\theta_{0}\right)\right)^{2} d H(\tau) \quad \text { for all } \theta \in M
$$


Hence we can pull this term out of the $\theta$-integral, giving

$$
\begin{aligned}
& \sum_{j=1}^{m} \frac{1}{\lambda_{j}(w)}-\sum_{j=1}^{m} \frac{1}{\lambda_{j}(v)} \\
& \quad \geq \int_{0}^{L} \int_{G} \sum_{j=1}^{m} \psi_{j}\left(x, \tau\left(\theta_{0}\right)\right)^{2} d H(\tau) \int_{M}[w(x, \theta)-v(x)] d V(\theta) d x
\end{aligned}
$$

from which (11.1) follows in view of the hypothesis (2.11) that the means of $w$ over $M$ are at least as large as those of $v$. The equality statement for (11.1) also follows, when $m \geq 2$, since $\psi_{1}^{2}$ is everywhere positive.

For the equality statement when $m=1$, note that (as in Section 13) the first eigenfunction $\psi_{1}(x, \theta)=\psi_{1}(x)$ is independent of $\theta \in M$, and so it is easy to use the hypothesis (2.11) to establish the inequalities in (13.8). The argument following (13.8) then yields the equality statement of (11.1) when $m=1$.

\section{Proof of Theorem 7 .}

Notice that if we define $v \equiv 1$, then all the hypotheses of Theorem 6 are satisfied except for (2.11). Hence we can mimic the proof of Theorem 6 in Section 11 up until the final step, where (2.11) is applied. By specializing (11.3) of that proof to $v \equiv 1$, we see that

$$
\begin{aligned}
& \sum_{j=1}^{m} \frac{1}{\lambda_{j}(w)}-\sum_{j=1}^{m} \frac{1}{\lambda_{j}(1)} \\
& \quad \geq \int_{0}^{L} \int_{G} \sum_{j=1}^{m} \psi_{j}\left(x, \tau\left(\theta_{0}\right)\right)^{2} d H(\tau) \int_{M}[w(x, \theta)-1] d V(\theta) d x
\end{aligned}
$$

and that our remaining tasks are: to show this last quantity is positive unless

$$
\int_{M} w(x, \theta) d V(\theta)=V(M) \quad \text { for almost every } x
$$

and to show that if $\lambda_{1}(w)=\lambda_{1}(1)$ then $w=1$ a.e. In proving all this, we can assume $\lambda_{m+1}(1)>\lambda_{m}(1)$, by the same reasoning as used in the proof of Theorem 4.

To start with, the eigenvalues $\left\{\lambda_{j}(1): j=1,2,3, \ldots\right\}$ can be computed by separation of variables to be

$$
\left\{\lambda_{k \ell}=\mu_{k}+(2 \ell-1)^{2}(\pi / 2 L)^{2}: k \geq 0, \ell \geq 1\right\},
$$


with corresponding eigenfunctions

$$
\psi_{k \ell}(x, \theta):=\sqrt{\frac{2}{L \lambda_{k \ell}}} \nu_{k}(\theta) \cos \left(\frac{(2 \ell-1) \pi x}{2 L}\right)
$$

satisfying $-\Delta \psi_{k \ell}=\lambda_{k \ell} \psi_{k \ell}$, where the $\nu_{k}$ are the eigenfunctions of $\Delta_{g}$ on $M$ (chosen to be orthonormal in $\mathcal{L}^{2}\left(M_{g}\right)$ ), with $-\Delta_{g} \nu_{k}=\mu_{k} \nu_{k}$. Note that $0=\mu_{0}<\mu_{1} \leq \mu_{2} \leq \cdots$. Now, $\left\{\psi_{k \ell}\right\}$ is a linearly independent set in $H_{m i x}^{1}((0, L) \times M)$ and it satisfies the orthonormality condition

$$
\int_{0}^{L} \int_{M} \nabla \psi_{k \ell} \cdot \nabla \psi_{k^{\prime} \ell^{\prime}} d V(\theta) d x=\delta_{k k^{\prime}} \delta_{\ell \ell^{\prime}}
$$

so that the $\psi_{k \ell}$ satisfy the conditions (11.2), with $v \equiv 1$.

Let $I:=\left\{(k, \ell): \lambda_{k \ell} \leq \lambda_{m}(1)\right\}$. Then $I$ contains exactly $m$ elements since $\lambda_{m}(1)<\lambda_{m+1}(1)$, and the numbers $\lambda_{k \ell}$ for $(k, \ell) \in I$ are a permutation of the eigenvalues $\lambda_{j}(1)$ for $j=1, \ldots, m$. Using the $\psi_{k \ell}$ in place of the $\psi_{j}$ in (12.1) and defining

$$
F(x):=\int_{M}[w(x, \theta)-1] d V(\theta) \quad \text { for } x \in(0, L)
$$

shows that we want to prove

$$
\int_{0}^{L} \int_{G} \sum_{(k, \ell) \in I} \psi_{k \ell}\left(x, \tau\left(\theta_{0}\right)\right)^{2} d H(\tau) F(x) d x>0
$$

unless $F(x)=0$ a.e.; we show separately later that if $\lambda_{1}(w)=\lambda_{1}(1)$ then $w=1$ a.e.

Recall that by hypothesis in the theorem, $F$ is decreasing. By changing $F$ on a set of measure 0 , we can assume that $F$ is right-continuous.

We can rewrite (12.5) as

$$
\int_{0}^{L} \sum_{\ell=1}^{\ell^{*}} \frac{2 \pi}{L} e_{\ell} \cos ^{2}\left(\frac{(2 \ell-1) \pi x}{2 L}\right) F(x) d x>0,
$$

where $\ell^{*}$ is the largest $\ell$ such that $I(\ell):=\{k \geq 0:(k, \ell) \in I\}$ is non-empty and

$$
e_{\ell}:=\frac{1}{\pi} \sum_{k \in I(\ell)} \frac{1}{\mu_{k}+(2 \ell-1)^{2}(\pi / 2 L)^{2}} \int_{G} \nu_{k}\left(\tau\left(\theta_{0}\right)\right)^{2} d H(\tau) .
$$


Using the double angle formula for $\cos ^{2}$ turns (12.6) into

$$
\begin{aligned}
\int_{0}^{L} \sum_{\ell=1}^{\ell^{*}} \frac{\pi}{L} e_{\ell} & \cos \left(\frac{(2 \ell-1) \pi x}{L}\right) F(x) d x \\
& +\sum_{\ell=1}^{\ell^{*}} \frac{\pi}{L} e_{\ell}\left[\int_{0}^{L} \int_{M} w(x, \theta) d V(\theta) d x-L \cdot V(M)\right]>0 .
\end{aligned}
$$

The second term is nonnegative by hypothesis, in this theorem, and is strictly positive unless

$$
\int_{0}^{L} F(x) d x=\int_{0}^{L} \int_{M} w(x, \theta) d V(\theta) d x-L \cdot V(M)=0 .
$$

We aim now to show that the first term in (12.7) is nonnegative and is in fact positive unless $F$ is constant. This will prove that (12.7) holds unless $F=0$ a.e. After integrating the first term in (12.7) by parts in $x$ it becomes

$$
-\int_{0}^{L} \sum_{\ell=1}^{\ell^{*}} \frac{e_{\ell}}{2 \ell-1} \sin \left(\frac{(2 \ell-1) \pi x}{L}\right) d F(x)
$$

and since $d F \leq 0$ by our hypothesis (2.13), it is enough now to show that

$$
\sum_{\ell=1}^{\ell^{*}} \frac{e_{\ell}}{2 \ell-1} \sin \left(\frac{(2 \ell-1) \pi x}{L}\right)>0 \quad \text { for all } x \in(0, L) \text {. }
$$

Obviously (12.8) follows from Lemma 15 if $\left\{e_{\ell}\right\}$ is decreasing. But $I(\ell+1) \subset$ $I(\ell)$ because of our definition of $I$, and so from the definition of $e_{\ell}$ we easily deduce that $\left\{e_{\ell}\right\}$ is indeed decreasing.

Lastly, suppose $\lambda_{1}(w)=\lambda_{1}(1)$. Then (12.7) must fail to hold for $m=1$ and so (by above) $F=0$ a.e, or $\int_{M} w(x, \theta) d V(\theta)=V(M)$ for almost all $x$. In particular, Theorem 8 applies (with $v \equiv 1$ ) and hence $w=1$ a.e., which is our desired equality statement in the case $m=1$.

\section{Proof of Theorem 8.}

The following proof is based on the proof of [17, Theorem 4], which deals with the ball under fixed boundary conditions.

Let $\psi_{1}$ be a first eigenfunction for $v^{-1} \Delta$ on $(0, L) \times M$, so that $-\Delta \psi_{1}=$ $\lambda_{1}(v) v \psi_{1}$ weakly. We may assume $\psi_{1}$ is positive because, as we remarked 
in Section $5, \psi_{1}$ is continuous and never zero. Also $\psi_{1}(x, \theta)=\psi_{1}(x)$ is independent of $\theta$, since the first eigenfunction for $v^{-1} \Delta$ is unique up to constant factors (see Section 5) and $\psi_{1}(x, \tau(\theta)$ ) is such a first eigenfunction for every isometry $\tau$ of $M$; use here that $v=v(x)$ is independent of $\theta \in M$ and that the isometry group on $M$ is transitive. Therefore $\psi_{1}(x)$ is positive and satisfies $-\psi_{1}^{\prime \prime}=\lambda_{1}(v) v \psi_{1}$ weakly in $H_{m i x}^{1}(0, L)$. Hence

$$
\psi_{1}(s)=-\lambda_{1}(v)(d / d s)^{-2}\left(v \psi_{1}\right)=\lambda_{1}(v) \int_{s}^{L} \int_{0}^{\rho} \psi_{1}(x) v(x) d x d \rho
$$

is $C^{1}$-smooth, with $\psi_{1}(L)=0$ and

$$
\psi_{1}^{\prime}(s)<0 \quad \text { for all } s \in(0, L) .
$$

By the Rayleigh principle, using $\psi_{1}$ as a trial function for $\lambda_{1}(w)$ yields that

$$
\lambda_{1}(w) \leq \frac{\int_{0}^{L} \int_{M}\left|\nabla \psi_{1}\right|^{2} d V(\theta) d x}{\int_{0}^{L} \int_{M} \psi_{1}^{2} w d V(\theta) d x} .
$$

And since $\psi_{1}(L)=0$, integration by parts in $s$ gives that

$$
\begin{aligned}
\int_{0}^{L} \int_{M} \psi_{1}^{2} w d V(\theta) d x & =\int_{0}^{L} \psi_{1}(s)^{2} \int_{M} w(s, \theta) d V(\theta) d s \\
& =-2 \int_{0}^{L} \psi_{1}(s) \psi_{1}^{\prime}(s) \int_{0}^{s} \int_{M} w(x, \theta) d V(\theta) d x d s \\
& \geq-2 \int_{0}^{L} \psi_{1}(s) \psi_{1}^{\prime}(s) \int_{0}^{s} \int_{M} v(x) d V(\theta) d x d s \\
& =\int_{0}^{L} \int_{M} \psi_{1}^{2} v d V(\theta) d x
\end{aligned}
$$

Therefore

$$
\lambda_{1}(w) \leq \frac{\int_{0}^{L} \int_{M}\left|\nabla \psi_{1}\right|^{2} d V(\theta) d x}{\int_{0}^{L} \int_{M} \psi_{1}^{2} w d V(\theta) d x} \leq \frac{\int_{0}^{L} \int_{M}\left|\nabla \psi_{1}\right|^{2} d V(\theta) d x}{\int_{0}^{L} \int_{M} \psi_{1}^{2} v d V(\theta) d x}=\lambda_{1}(v)
$$

which is the inequality we want. 
If $\lambda_{1}(w)=\lambda_{1}(v)$ then equality reigns in (13.8) and so $\psi_{1}$ is a $\lambda_{1}(w)$ eigenfunction for $w^{-1} \Delta$, as well as being a $\lambda_{1}(v)$-eigenfunction for $v^{-1} \Delta$. Hence

$$
\psi_{1} w=\frac{-\Delta \psi_{1}}{\lambda_{1}(w)}=\frac{-\Delta \psi_{1}}{\lambda_{1}(v)}=\psi_{1} v
$$

weakly in $H_{m i x}^{1}(0, L)$, and thus $\psi_{1} w=\psi_{1} v$ a.e. Because $\psi_{1}$ is positive, $w=v$ a.e. as claimed.

\section{Proof of Theorem 11.}

Once more, we start by reducing to the case $\Phi=$ id. Fix $n \geq 1$. (For the theorem itself we take $n=4$, but for the remarks following the theorem we want to consider arbitrary $n$.) Assume for a moment that we know

$$
\sum_{j=1}^{m} \frac{1}{\lambda_{j}(w)} \geq \sum_{j=1}^{m} \frac{1}{\lambda_{j}(1)}
$$

for each $m$ with $1 \leq m \leq n$, with the inequality being strict when $m \geq 2$ unless $\int_{0}^{2 \pi}[w(x, \theta)+w(-x, \theta)] d \theta=4 \pi$ for almost all $x \in(0, L)$, and being strict when $m=1$ unless $w=1$ a.e. Then (3.3) and its equality statements follow, by exactly the same argument as in the first paragraph of Section 10.

Thus we have only to prove (14.1) and its equality statements. The case $m=1$ is a special case of Theorem 14 (with $v \equiv 1, M=S^{1}$ ), which we prove in Section 17, and so we can fix $m$ with $2 \leq m \leq n$ from now on.

We begin to prove (14.1) by describing the eigenvalues and eigenfunctions of $(-L, L) \times S^{1}$, with $w \equiv 1$. The eigenvalues $\left\{\lambda_{j}(1): j=1,2,3, \ldots\right\}$ can be computed by separation of variables to be

$$
\left\{\lambda_{k \ell}=k^{2}+(\ell \pi / 2 L)^{2}: k \in \mathbf{Z}, \ell \geq 1\right\},
$$

with corresponding normalized eigenfunctions

$\psi_{k \ell}(x, \theta):=\frac{1}{\sqrt{\pi L \lambda_{k \ell}}} \times\left\{\begin{array}{ll}\cos \left(\frac{\ell \pi x}{2 L}\right), & \text { if } \ell \text { is odd } \\ \sin \left(\frac{\ell \pi x}{2 L}\right), & \text { if } \ell \text { is even }\end{array}\right\} \times\left\{\begin{array}{ll}\sin k \theta, & \text { if } k>0 \\ 1 / \sqrt{2}, & \text { if } k=0 \\ \cos k \theta, & \text { if } k<0\end{array}\right\}$

satisfying $-\Delta \psi_{k \ell}=\lambda_{k \ell} \psi_{k \ell}$. Then $\left\{\psi_{k \ell}\right\}$ is a linearly independent set in $H_{0}^{1}\left((-L, L) \times S^{1}\right)$ and it satisfies the orthonormality condition

$$
\int_{-L}^{L} \int_{0}^{2 \pi} \nabla \psi_{k \ell} \cdot \nabla \psi_{k^{\prime} \ell^{\prime}} d \theta d x=\delta_{k k^{\prime}} \delta_{\ell \ell^{\prime}}
$$


Let $I$ be a collection of $m$ distinct ordered pairs $(k, \ell)$ such that the numbers $\lambda_{k \ell}$ for $(k, \ell) \in I$ are a permutation of the eigenvalues $\lambda_{j}(1)$ for $1 \leq j \leq m$. (This set $I$ need not be unique.)

By using the $\psi_{k \ell}$ as trial functions in the variational characterization for $\sum_{j=1}^{m} \lambda_{j}(w)^{-1}$ (for which adapt (5.2)), we obtain that

$$
\begin{aligned}
\sum_{j=1}^{m} \frac{1}{\lambda_{j}(w)} \geq \sum_{(k, \ell) \in I} \int_{-L}^{L} \int_{0}^{2 \pi}\left|\psi_{k \ell}\right|^{2} w d \theta d x & \\
= & \sum_{(k, \ell) \in I} \frac{1}{\pi L \lambda_{k \ell}} \int_{-L}^{L} \int_{0}^{2 \pi}\left\{\begin{array}{ll}
\cos ^{2}\left(\frac{\ell \pi x}{2 L}\right), & \text { if } \ell \text { is odd } \\
\sin ^{2}\left(\frac{\ell \pi x}{2 L}\right), & \text { if } \ell \text { is even }
\end{array}\right\} \\
& \times\left\{\begin{array}{ll}
\sin ^{2} k \theta, & \text { if } k>0 \\
1 / 2, & \text { if } k=0 \\
\cos ^{2} k \theta, & \text { if } k<0
\end{array}\right\} w(x, \theta) d \theta d x
\end{aligned}
$$

The same argument holds if we replace $I$ by $\{(k, \ell):(-k, \ell) \in I\}$, and averaging the two resulting inequalities gives

$$
\begin{aligned}
& \sum_{j=1}^{m} \frac{1}{\lambda_{j}(w)} \\
& \geq \sum_{(k, \ell) \in I} \frac{1}{2 \pi L \lambda_{k \ell}} \int_{-L}^{L}\left\{\begin{array}{ll}
\cos ^{2}\left(\frac{\ell \pi x}{2 L}\right), & \text { if } \ell \text { is odd } \\
\sin ^{2}\left(\frac{\ell \pi x}{2 L}\right), & \text { if } \ell \text { is even }
\end{array}\right\} \int_{0}^{2 \pi} w(x, \theta) d \theta d x \\
& =\sum_{(k, \ell) \in I} \frac{1}{2 \pi L \lambda_{k \ell}} \int_{0}^{L}\left\{\begin{array}{ll}
\cos ^{2}\left(\frac{\ell \pi x}{2 L}\right), & \text { if } \ell \text { is odd } \\
\sin ^{2}\left(\frac{\ell \pi x}{2 L}\right), & \text { if } \ell \text { is even }
\end{array}\right\} \\
& \quad \times \int_{0}^{2 \pi}[w(x, \theta)+w(-x, \theta)] d \theta d x \\
& =\sum_{(k, \ell) \in I} \frac{\ell(-1)^{\ell+1}}{4 L^{2} \lambda_{k \ell}} \int_{0}^{L} \sin (\ell \pi s / L) \int_{-s}^{s} \int_{0}^{2 \pi} w(x, \theta) d x d s \text { by parts in } x \\
& =\sum_{(k, \ell) \in I} \frac{\ell}{4 L^{2} \lambda_{k \ell}} \int_{0}^{L} \sin (\ell \pi(L-s) / L) \int_{-s}^{s} \int_{0}^{2 \pi} w(x, \theta) d x d s .
\end{aligned}
$$

We show below that

$$
g(y):=\sum_{(k, \ell) \in I} \frac{\ell}{\lambda_{k \ell}} \sin (\ell y)>0 \quad \text { for all } y \in(0, \pi),
$$


provided $m \leq 4$, and so from the hypothesis $\int_{-s}^{s} \int_{0}^{2 \pi} w(x, \theta) d \theta d x \geq 2 \pi \cdot 2 s$ in the theorem it follows that

$$
\begin{aligned}
\sum_{j=1}^{m} \frac{1}{\lambda_{j}(w)} & \geq \sum_{(k, \ell) \in I} \frac{\ell \pi}{L^{2} \lambda_{k \ell}} \int_{0}^{L} \sin (\ell \pi(L-s) / L) s d s \\
& =\sum_{(k, \ell) \in I} \frac{1}{\lambda_{k \ell}}=\sum_{j=1}^{m} \frac{1}{\lambda_{j}(1)},
\end{aligned}
$$

giving (14.1) for $n=4$. If equality holds in (14.1), then the above argument gives that $\int_{-s}^{s} \int_{0}^{2 \pi} w(x, \theta) d \theta d x=2 \pi \cdot 2 s$ for all $s$, and so $\int_{0}^{2 \pi}[w(x, \theta)+$ $w(-x, \theta)] d \theta=4 \pi$ for almost all $x$, as desired.

We must still prove (14.2). We begin by proving it when $I$ lies on the union of the $\ell$-axis and the line $\ell=1$, that is, we assume $I$ has the property

$$
(k, \ell) \in I \quad \Longrightarrow \quad \text { either } k=0 \text { or } \ell=1 \text {. }
$$

Note also that by the definition of $I$, if $(0, \ell) \in I$ and $1 \leq \ell^{\prime}<\ell$ then $\left(0, \ell^{\prime}\right) \in I$. Hence we can write

$$
g(y)=\left(\frac{2 L}{\pi}\right)^{2} \sum_{\ell=1}^{\ell^{*}} \frac{\sin (\ell y)}{\ell}+c \sin (y)
$$

for some $\ell^{*} \geq 1$ and some nonnegative constant $c$. Since $\sum_{\ell=1}^{\ell^{*}} \ell^{-1} \sin (\ell y)$ is positive for $y \in(0, \pi)$ by [29, p. 62], we have proved (14.2) in this case.

Next, from the definition of $I$ it is clear that

$$
(k, \ell) \in I, \quad \lambda_{k^{\prime} \ell^{\prime}}<\lambda_{k \ell} \quad \Longrightarrow \quad\left(k^{\prime}, \ell^{\prime}\right) \in I .
$$

Consequently when $m \leq 4$, the set $I$ cannot contain $( \pm 1,2)$, and so it lies on the union of the $\ell$-axis and the line $\ell=1$. Thus (14.2) holds when $m \leq 4$, which proves (14.1) for $n \leq 4$; this proves the theorem.

It remains to justify the remarks made after the theorem. From now on, then, assume $n \geq m \geq 5$. The existence of the numbers $L_{1}(n)$ and $L_{2}(n)$ is easy to explain: if $L$ is sufficiently close to 0 then $I$ must lie on the line $\ell=1$, and if $L$ is sufficiently close to $+\infty$ then $I$ must lie entirely on the $\ell$-axis, with $k=0$. In both cases (14.2) holds and we are done. Of course, it might be possible to improve upon the values of $L_{1}$ and $L_{2}$ found this way, and this we do for $L_{1}(5)$ below.

We must still establish the claimed values of $L_{1}(5)$ and $L_{2}(5)$, for $n=5$. We have considered $m \leq 4$ above, and so we need only consider $m=5$ and 
show that (14.2) holds so long as $L \leq \sqrt{2} \pi / 2$ or $L \geq \sqrt{5} \pi / 2$. Write

$$
z:=\left(\frac{\pi}{2 L}\right)^{2}
$$

so that $\lambda_{k, \ell}=k^{2}+\ell^{2} z$. If $L \in[\sqrt{5} \pi / 2,+\infty$ ) (so that $z \leq 1 / 5$ ) then $\lambda_{0,3}=9 z \leq 1+4 z=\lambda_{1,2}$ and so $I$ can be chosen to lie on the union of the $\ell$-axis and the line $\ell=1$ (since $I$ has only 5 elements), so that (14.2) holds. This shows that we can take $L_{2}(5)=\sqrt{5} \pi / 2$. Now assume $L \in(0, \sqrt{2} \pi / 2]$ (so that $z \geq 1 / 2$ ). Notice that we can suppose either $(1,2) \in I$ or $(-1,2) \in I$, since otherwise $I$ would lie on the union of the $\ell$-axis and the line $\ell=1$ and we would be done. By symmetry we can assume $(1,2) \in I$. Then

$$
I=\{(0,1),(1,1),(-1,1),(0,2),(1,2)\}
$$

and for $y \in(0, \pi)$ we have

$$
\begin{aligned}
g(y) & =\left\{\frac{1}{z}+\frac{2}{1+z}\right\} \sin y+\left\{\frac{2}{4 z}+\frac{2}{1+4 z}\right\} \sin (2 y) \\
& =\left\{\frac{1}{z}+\frac{2}{1+z}+\left(\frac{1}{z}+\frac{4}{1+4 z}\right) \cos y\right\} \sin y \\
& >\left\{\frac{1}{z}+\frac{2}{1+z}-\left(\frac{1}{z}+\frac{4}{1+4 z}\right)\right\} \sin y \\
& \geq 0
\end{aligned}
$$

(since $z \geq 1 / 2$ ), and from this it follows that we can take $L_{1}(5)=\sqrt{2} \pi / 2$.

\section{Proof of Theorem 12.}

Simply modify the proof of Theorem 6 in Section 11 . Note that now $x \in$ $(-L, L)$ and so we integrate $\int_{-L}^{L}$ rather than $\int_{0}^{L}$, for example. Furthermore, the $\psi_{j}$ should now belong to $H_{0}^{1}((-L, L) \times M)$ rather than to $H_{m i x}^{1}((0, L) \times$ $M)$.

For later reference, the analogue of (11.3) that we obtain is:

$$
\begin{aligned}
& \sum_{j=1}^{m} \frac{1}{\lambda_{j}(w)}-\sum_{j=1}^{m} \frac{1}{\lambda_{j}(v)} \\
& \quad \geq \int_{-L}^{L} \int_{G} \sum_{j=1}^{m} \psi_{j}\left(x, \tau\left(\theta_{0}\right)\right)^{2} d H(\tau) \int_{M}[w(x, \theta)-v(x)] d V(\theta) d x
\end{aligned}
$$




\section{Proof of Theorem 13.}

We prove the theorem by making the following changes to the proof of Theorem 7 in Section 12. Write:

Theorem 12

(3.4)

specializing (15.1)

$$
\begin{aligned}
& \int_{-L}^{L} \\
& {[w(x, \theta)+w(-x, \theta)] / 2} \\
& H_{0}^{1}((-L, L) \times M)
\end{aligned}
$$

instead of Theorem 6, instead of (2.11), instead of specializing (11.3), instead of $\int_{0}^{L}$ in (12.1), (12.3) and (11.2), instead of $w(x, \theta)$ in (12.2), instead of $H_{m i x}^{1}((0, L) \times M)$,

and in (12.4) define

$$
F(x):=\int_{M}[w(x, \theta)+w(-x, \theta)-2] d V(\theta)
$$

instead of $F(x):=\int_{M}[w(x, \theta)-1] d V(\theta)$, using also for (12.5) the evenness of $\psi_{k \ell}^{2}$ in $x$ (see below).

Furthermore, the eigenvalues should now be $\left\{\lambda_{k \ell}=\mu_{k}+(\ell \pi / 2 L)^{2}: k \geq\right.$ $0, \ell \geq 1\}$, with corresponding eigenfunctions

$$
\psi_{k \ell}(x, \theta):=\frac{1}{\sqrt{L \lambda_{k \ell}}} \nu_{k}(\theta) \times\left\{\begin{array}{ll}
\cos \left(\frac{\ell \pi x}{2 L}\right), & \text { if } \ell \text { is odd } \\
\sin \left(\frac{\ell \pi x}{2 L}\right), & \text { if } \ell \text { is even }
\end{array}\right\} .
$$

Also, we replace the material from (12.6) onwards with the following:

We can rewrite (12.5) as

$$
\int_{0}^{L} \sum_{\ell=1}^{\ell^{*}} \frac{2 \pi}{L} e_{\ell}\left\{\begin{array}{ll}
\cos ^{2}\left(\frac{\ell \pi x}{2 L}\right), & \text { if } \ell \text { is odd } \\
\sin ^{2}\left(\frac{\ell \pi x}{2 L}\right), & \text { if } \ell \text { is even }
\end{array}\right\} F(x) d x>0,
$$

where $\ell^{*}$ is the largest $\ell$ such that $I(\ell):=\{k \geq 0:(k, \ell) \in I\}$ is non-empty and

$$
e_{\ell}:=\frac{1}{2 \pi} \sum_{k \in I(\ell)} \frac{1}{\mu_{k}+(\ell \pi / 2 L)^{2}} \int_{G} \nu_{k}\left(\tau\left(\theta_{0}\right)\right)^{2} d H(\tau) .
$$

Using the double angle formulas for $\cos ^{2}$ and $\sin ^{2}$ turns (16.1) into

$$
\begin{aligned}
\int_{0}^{L} \sum_{\ell=1}^{\ell^{*}} \frac{\pi}{L} e_{\ell}(-1)^{\ell+1} \cos \left(\frac{\ell \pi x}{L}\right) F(x) d x \\
+\sum_{\ell=1}^{\ell^{*}} \frac{\pi}{L} e_{\ell}\left[\int_{-L}^{L} \int_{M} w(x, \theta) d V(\theta) d x-2 L \cdot V(M)\right]>0 .
\end{aligned}
$$


The second term is nonegative by hypothesis, in this theorem, and is strictly positive unless

$$
\int_{0}^{L} F(x) d x=\int_{-L}^{L} \int_{M} w(x, \theta) d V(\theta) d x-2 L \cdot V(M)=0 .
$$

We aim now to show that the first term in (16.2) is nonnegative and is in fact positive unless $F$ is constant. This will prove that (16.2) holds unless $F=0$ a.e.; we show later that if $\lambda_{1}(w)=\lambda_{1}(1)$ then $w=1$ a.e. After integrating the first term in (16.2) by parts in $x$ it becomes

$$
-\int_{0}^{L} \sum_{\ell=1}^{\ell^{*}} \frac{e_{\ell}}{\ell} \sin \left(\frac{\ell \pi(L-x)}{L}\right) d F(x) .
$$

Since $d F \leq 0$ by our hypothesis (3.6), to establish that this quantity is nonnegative (and is positive unless $F$ is constant) it is enough to show

$$
\sum_{\ell=1}^{\ell^{*}} \frac{e_{\ell}}{\ell} \sin \left(\frac{\ell \pi(L-x)}{L}\right)>0 \quad \text { for all } x \in(0, L) .
$$

Obviously (16.3) follows from Lemma 16 so long as $\left\{e_{\ell}\right\}$ is decreasing. But $I(\ell+1) \subset I(\ell)$ because of our definition of $I$, and so from the definition of $e_{\ell}$ we easily deduce that $\left\{e_{\ell}\right\}$ is indeed decreasing.

Lastly, suppose $\lambda_{1}(w)=\lambda_{1}(1)$. Then (16.2) must fail to hold for $m=1$ and so (by above) $F=0$ a.e., or $\int_{M}[w(x, \theta)+w(-x, \theta)] d V(\theta)=2 V(M)$ for almost all $x$. In particular, Theorem 14 applies (with $v \equiv 1$ ) and hence $w=1$ a.e., which is our desired equality statement in the case $m=1$.

\section{Proof of Theorem 14.}

To prove the theorem, adapt the proof of Theorem 8 in Section 13; simply write:

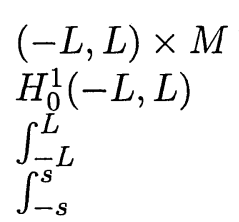

$(3.8)$ instead of
instead of
instead of
instead of
instead of

$(0, L) \times M$,

$H_{m i x}^{1}(0, L)$

$\int_{0}^{L}$ in (13.3), (13.4), (13.7) and (13.8),

$\int_{0}^{s}$ in (13.5) and (13.6),

(2.15) in (13.6).

Lastly, observe that $\psi(x)$ is even in $x$, because $v(x)$ is assumed to be even and the first eigenfunction for $v^{-1} \Delta$ is unique up to constant factors. 


\section{Appendix A. Summary of results and boundary conditions.}

Here we summarize the hypotheses and conclusions of the theorems both in this paper and in the related work [17]. We hope this gives the serious reader a clearer understanding of the structure of these papers.

This paper examines inhomogeneous strings and cylinders, under either mixed Dirichlet-Neumann boundary conditions (the "fixed-free") case or purely Dirichlet boundary conditions (the "fixed" case). For most of the results, the extremal is the homogeneous string or cylinder with mass density identically 1 . Our hypotheses on $w$ ensure, in the fixed-free case, that $w$ has more mass than 1 does near the free end of the string/cylinder, and in the fixed case, that $w$ has more mass than 1 near the middle. Specifically, we use three different kinds of hypotheses, for the fixed-free cylinder $(0, L) \times S^{1}$ :

(H1) "more mass on subcylinders":

$$
\int_{0}^{s} \int_{0}^{2 \pi} w(x, \theta) d \theta d x \geq 2 \pi s \quad \text { for all } s \in(0, L)
$$

(H2) "more mass on circles":

$$
\int_{0}^{2 \pi} w(x, \theta) d \theta \geq 2 \pi \quad \text { for all } x \in(0, L)
$$

(H3) "decreasing mass on circles":

$$
x \mapsto \int_{0}^{2 \pi} w(x, \theta) d \theta \quad \text { is decreasing for } x \in(0, L)
$$

$$
\text { and } \int_{0}^{L} \int_{0}^{2 \pi} w(x, \theta) d \theta d x \geq 2 \pi L .
$$

In each hypothesis, $w$ has more mass near the free end $x=0$ than does the mass density identically 1 . It is easy to check that $(\mathrm{H} 2) \Rightarrow(\mathrm{H} 1)$ and $(\mathrm{H} 3) \Rightarrow(\mathrm{H} 1)$ but not conversely, and that (H2) and (H3) are not comparable. Observe also that the hypotheses can be adapted to the cylinder with fixed boundary conditions (see Theorems 11, 12 and 13), and to strings (see Theorems 2 and 9, which use a "mass on subintervals" variant of (H1)).

Table 1 classifies the theorems in this paper according to their domain, boundary conditions, type of hypotheses and conclusions. In the second column of Table 1, "gen. cylinder" refers to a generalized cylinder $(0, L) \times M$, where $M$ is a compact homogeneous Riemannian manifold (so that $M$ has 
Eigenvalues of strings and cylinders with variable mass density

\begin{tabular}{|c|c|c|c|c|}
\hline & Domain & $B C s$ & Hypothesis & Conclusion \\
\hline Theorem 1 & interval & fixed-free & $\mathrm{H} 1$ & first eigenvalue \\
\hline Theorem 2 & interval & fixed-free & $\mathrm{H} 1$ & $\Phi$-functional, all $n$ \\
\hline Corollary 3 & interval & fixed-free & $\mathrm{H} 1$ & zeta function \\
\hline Theorem 4 & cylinder & fixed-free & $\mathrm{H} 1$ & $\Phi$-functional, all $n$ \\
\hline Comp. Result 5 & gen. cylinder & fixed-free & $\mathrm{H} 1$ & $\begin{array}{r}\Phi \text {-functional, } \\
n<53055\end{array}$ \\
\hline Theorem 6 & gen. cylinder & fixed-free & $\mathrm{H} 2$ & $\Phi$-functional, all $n$ \\
\hline Theorem 7 & gen. cylinder & fixed-free & $\mathrm{H} 3$ & $\Phi$-functional, all $n$ \\
\hline Theorem 8 & gen. cylinder & fixed-free & $\mathrm{H} 1$ & first eigenvalue \\
\hline Theorem 9 & interval & fixed & H1 & $\Phi$-functional, all $n$ \\
\hline Corollary 10 & interval & fixed & H1 & zeta function \\
\hline Theorem 11 & cylinder & fixed & $\mathrm{H} 1$ & $\Phi$-functional, $n \leq 4$ \\
\hline Theorem 12 & gen. cylinder & fixed & $\mathrm{H} 2$ & $\Phi$-functional, all $n$ \\
\hline Theorem 13 & gen. cylinder & fixed & $\mathrm{H} 3$ & $\Phi$-functional, all $n$ \\
\hline Theorem 14 & gen. cylinder & fixed & $\mathrm{H} 1$ & first eigenvalue \\
\hline
\end{tabular}

Table 1: Summary of results in this paper.

\begin{tabular}{l|l|c|c|c} 
& Domain & BCs & Hypothesis & Conclusion \\
\hline$[17$, Theorem 1] & ball & fixed & H1 & zeta function \\
{$[17$, Theorem 2] } & interval, dim. 1 & fixed & $\mathrm{H} 1$ & $\Phi$-functional, all $n$ \\
{$[17$, Theorem 3] } & ball, dim. $\geq 2$ & fixed & $\mathrm{H} 1$ & $\Phi$-functional, \\
& & fixed & $\mathrm{H} 1$ & first eigenvalue \\
{$[17$, Theorem 4] } & ball & fixed & curvature $\geq 0$ & zeta function
\end{tabular}

Table 2: Summary of results in [17].

transitive isometry group). When $M$ is a circle this is just the standard cylinder.

Table 2 similarly classifies the theorems in the related paper [17]. Note that Theorem 9 and Corollary 10 of this paper are directly quoted from [17].

\section{References.}

[1] M. S. Ashbaugh and R. D. Benguria, Isoperimetric inequalities for eigenvalue ratios, In: Partial Differential Equations of Elliptic Type, pp. 1-36, Editors: A. Alvino, E. Fabes and G. Talenti, Cambridge, Cambridge University Press 1994.

[2] C. Bandle, Isoperimetric Inequalities and Applications, Pitman, London 1980. 
[3] D. Banks, Bounds for the eigenvalues of some vibrating systems, Pacific J. Math, 10 (1960), 439-474.

[4] P. R. Beesack and B. Schwarz, On the zeros of solutions of second order linear differential equations, Canad. J. Math, 8 (1956), 504-515.

[5] P. H. Bérard, Spectral Geometry: Direct and Inverse Problems, Lecture Notes in Mathematics, 1207, Springer-Verlag, Berlin 1986.

[6] W. M. Boothby, An Introduction to Differentiable Manifolds and Riemannian Geometry, Second edition, Academic Press, San Diego 1986.

[7] T. P. Branson and P. B. Gilkey, The asymptotics of the Laplacian on a manifold with boundary, Comm. in PDE, 15 (1990), 245-272.

[8] H. J. Brascamp, E. H. Lieb and J. M. Luttinger, A general rearrangement inequality for multiple integrals, J. Funct. Anal., 17 (1974), 227-237.

[9] S.-Y. A. Chang, On zeta functional determinant, In: Partial Differential Equations and their Applications, pp. 25-50, CRM Proc. Lecture Notes, 12, American Mathematical Society, Providence, RI 1997.

[10] I. Chavel, Eigenvalues in Riemannian Geometry, Academic Press, Orlando, Florida 1984.

[11] M. Engman, Trace formulae for $S^{1}$ invariant Green's operators on $S^{2}$, Manuscripta Math., 93 (1997), 357-368.

[12] D. Gilbarg and N. S. Trudinger, Elliptic Partial Differential Equations of Second Order, Springer-Verlag, Berlin 1983.

[13] I. S. Gradshteyn and I. M. Ryzhik, Table of Integrals, Series and Products, Fifth edition, Editor, A. Jeffrey, Translated from the Russian by Scripta Technica, Inc. Academic Press, Boston 1994.

[14] J. Hersch, Anwendungen der konformen Abbildung auf isoperimetrische Sätze für Eigenwerte, In: Festband zum 70. Geburtstag von Rolf Nevanlinna, pp. 2534, Springer-Verlag, Berlin 1966.

[15] S. Kobayashi and K. Nomizu, Foundations of Differential Geometry, Volume I, Wiley-Interscience, New York 1963.

[16] R. S. Laugesen, Eigenvalues of Laplacians with mixed boundary conditions, under conformal mapping, Illinois J. Math., 42 (1998), 19-39.

[17] R. S. Laugesen, Eigenvalues of the Laplacian on inhomogeneous membranes, Amer. J. Math., 120 (1998), 305-344. 
[18] R. S. Laugesen and C. Morpurgo, Extremals for eigenvalues of Laplacians under conformal mapping, J. Funct. Anal., 155 (1), 64-108, 1998.

[19] J. M. Luttinger, Generalized isoperimetric inequalities, I-III, J. Math. Phys., 14 (1973), 586-593, 14 (1973), 1444-1447, 14 (1973), 1448-1450. (See [8] for an elegant proof of the rearrangement result Luttinger uses.)

[20] H. L. Montgomery, Minimal theta functions, Glasgow Math. J., 30 (1988), $75-85$.

[21] C. Morpurgo, Local extrema of traces of heat kernels on $S^{2}$, J. Funct. Anal., 141 (1996), 335-364.

[22] C. Morpurgo, The logarithmic Hardy-Littlewood-Sobolev inequality and extremals of zeta functions on $S^{n}$, Geom. Funct. Anal. (GAFA), 6 (1996), 146171.

[23] Z. Nehari, On the principal frequency of a membrane, Pacific J. Math., 8 (1958), 285-293.

[24] B. Osgood, R. Phillips and P. Sarnak, Extremals of determinants of Laplacians, J. Funct. Anal., 80 (1988), 148-211.

[25] G. Pólya and G. Szegö, Isoperimetric Inequalities in Mathematical Physics, Princeton University Press, Princeton 1951.

[26] B. Schwarz, Bounds for the principal frequency of the nonhomogeneous membrane and for the generalized Dirichlet integral, Pacific J. Math., 7 (1957), 1653-1676.

[27] B. Schwarz, Bounds for sums of reciprocals of eigenvalues, Bull. Res. Counc. of Israel, F, 8 (1959), 91-102.

[28] H. F. Weinberger, A First Course in Partial Differential Equations, John Wiley, New York 1965.

[29] A. Zygmund, Trigonometric Series, Volume I, Cambridge University Press, Cambridge 1988.

RECEIVED APRIL 1, 1998.

UNIVERSITY OF ILLINOIS,

URBANA, IL 61801,

U.S.A.

E-mail address: laugesen@math.uiuc.edu 